Die Schweiz und die humanitäre Aktion

\title{
8. Zusammenarbeit mit den Entwicklungsländern
}

\section{Gérard Perroulaz}

\section{(2) OpenEdition}

\section{Journals}

Electronic version

URL: http://journals.openedition.org/sjep/666

ISSN: 1663-9677

Publisher

Institut de hautes études internationales et du développement

Printed version

Date of publication: 1 janvier 1999

Number of pages: 227-255

ISSN: $1660-5926$

\section{Electronic reference}

Gérard Perroulaz, « 8. Zusammenarbeit mit den Entwicklungsländern », Schweizerisches Jahrbuch für Entwicklungspolitik [Online], 18 | 1999, Online erschienen am: 28 August 2012, abgerufen am 07 September 2020. URL : http://journals.openedition.org/sjep/666 


\section{ZUSAMMENARBEIT MIT DEN ENTWICKLUNGSLÄNDERN*}

\subsection{ALLGEMEINE TENDENZEN DER ÖFFENTLICHEN ENTWICKLUNGSHILFE}

Der Ausschuss für Entwicklungshilfe (DAC) ist das Organ der OECD, das sich mit Fragen der Entwicklungszusammenarbeit befasst. Die Mitgliedsländer beraten sich, um das Volumen und die Art ihrer Entwicklungshilfebeiträge zu untersuchen. Sie prüfen periodisch (alle drei Jahre) die Entwicklungshilfeprogramme jedes der Mitgliedsländer. Auch stimmen sie sich untereinander zur Ausarbeitung gemeinsamer Strategien im Kooperationsbereich ab.

\section{$\square$ Rückläufiger Trend der Hilfe der DAC-Länder}

Die gesamte öffentliche Entwicklungshilfe ist in den letzten Jahren stark zurückgegangen. Sie ist von 60,9 Milliarden Dollar 1992 auf 55,4 Milliarden Dollar 1996 bzw. 47,6 Milliarden Dollar 1997 gesunken (siehe Tabelle 18). Die Vereinten Nationen empfehlen den Geberländern, $0,7 \%$ ihres Bruttosozialprodukts (BSP) für die öffentliche Entwicklungshilfe aufzubringen, jedoch liegen nur Dänemark, Norwegen, die Niederlande und Schweden über diesem Ziel. Die öffentliche Entwicklungshilfe sämtlicher DAC-Länder machte 1997 nur noch $0,22 \%$ des BSP aus und erreichte somit den niedrigsten Prozentsatz, seitdem das Ziel von 0,7\% festgesetzt wurde. Der gewogene Mittelwert der DAC-Staaten lag 1992 noch bei 0,33\% des BSP, und die öffentliche Entwicklungshilfe hatte sich zwei Jahrzehnte lang, bis Anfang der 90er Jahre bei 0,35\% des BSP gehalten.

\section{Öffentliche Hilfe für Entwicklungsländer und Hilfe für Transitionsländer Neueste Änderungen der Liste der Empfängerländer}

Das DAC unterscheidet seit 1994 zwei Länderlisten, die der Entwicklungsländer und die der Transitionsländer*.

Die Hilfe für die Entwicklungsländer und -gebiete wird unter dem Aggregat "öffentliche Entwicklungszusammenarbeit" verbucht. Bestimmte Länder Mittel- und Osteuropas sind auf der Liste der Entwicklungsländer aufgeführt, und die Hilfe für diese Länder kann somit in der öffentlichen Entwicklungshilfe verbucht werden. Es handelt sich um Albanien, die Nachfolgestaaten des früheren Jugoslawiens, die zentralasiatischen Staaten (Armenien, Aserbeidschan, Kasachstan, Kirgistan, Usbekistan, Tadschikistan, Turkmenistan) sowie Georgien und Moldawien (Moldawien ist seit 1997 auf die Liste der Entwicklungsländer versetzt worden).

Die zweite Liste umfasst die Transitionsländer und -gebiete, das heisst die mittel- und osteuropäischen Staaten, die Länder der Gemeinschaft Unabhängiger Staaten, aber auch - was seit einigen Jahren neu eingeführt wurde - gewisse weiter fortgeschrittene Entwicklungsländer**. Die Leistungen für diese Länder sind nicht in der öffentlichen Entwicklungshilfe, sondern in einem separaten Aggregat "öffentliche Hilfe für die Transitionsländer» enthalten. Seit 1997 wurde als wichtige Neuheit eingeführt, dass die Hilfe für Israel nicht mehr in der öffentlichen Entwicklungszusammenarbeit (EZA) verbucht werden kann (wohingegen die Hilfe für die Gebiete unter palästinensischer Verwaltung in der öffentlichen EZA

* Von Gérard Perroulaz, Forschungsbeauftragter am IUED, Genf. 
verbucht wird). Somit ist diesen Änderungen bei einem Vergleich der Entwicklung der öffentlichen Entwicklungshilfe seit Anfang der 90er Jahre stets Rechnung zu tragen. Zum Beispiel lassen sich zwei Drittel des Rückgangs der öffentlichen Entwicklungszusammenarbeit der USA zwischen 1996 und 1997 durch die Streichung Israels von der Liste der Entwicklungsländer erklären.

$\llbracket$ JSDW 1999, die Hilfe für die Transitionsländer wird im Kapitel 9 der "Jahresübersicht» des Jahrbuchs ausführlich beschrieben. * Die beiden vollständigen DAC-Listen der Hilfeempfängerländer sind am Ende der «Jahresübersicht« aufgeführt.

** Liste der fortgeschritteneren Entwicklungsländer, deren Hilfe nicht mehr in der öffentlichen Entwicklungshilfe berücksichtigt werden kann: Bahamas, Bermuda-Inseln, Brunei, Cayman-Inseln, Zypern, Vereinigte Arabische Emirate, Falkland-Inseln, Hongkong, Israel, Katar, Singapur, Taiwan.

Tabelle Nr. 18: Öffentliche Entwicklungshilfe der DAC-Länder von 1992 bis 1997 (in \% des BSP)

\begin{tabular}{lrrrrrrl}
\hline & $\mathbf{1 9 9 2}$ & $\mathbf{1 9 9 3}$ & $\mathbf{1 9 9 4}$ & $\mathbf{1 9 9 5}$ & $\mathbf{1 9 9 6}$ & $\mathbf{1 9 9 7}$ & Tendenz \\
\hline Australien & 0.37 & 0.35 & 0.34 & 0.36 & 0.30 & 0.28 & Rückgang \\
\hline Österreich & 0.30 & 0.30 & 0.33 & 0.33 & 0.24 & 0.26 & Rückgang \\
\hline Belgien & 0.39 & 0.39 & 0.32 & 0.38 & 0.34 & 0.31 & Rückgang \\
\hline Kanada & 0.46 & 0.45 & 0.43 & 0.38 & 0.32 & 0.36 & Rückgang \\
\hline Dänemark & 1.02 & 1.03 & 1.03 & 0.96 & 1.04 & 0.97 & Gleichbleibend* \\
\hline Finnland & 0.64 & 0.45 & 0.31 & 0.32 & 0.34 & 0.33 & Starker Rückgang \\
\hline Frankreich & 0.63 & 0.63 & 0.64 & 0.55 & 0.48 & 0.45 & Rückgang \\
\hline Deutschland & 0.39 & 0.36 & 0.34 & 0.31 & 0.33 & 0.28 & Rückgang \\
\hline Irland & 0.16 & 0.20 & 0.25 & 0.29 & 0.31 & 0.31 & Steigerung \\
\hline Italien & 0.34 & 0.31 & 0.27 & 0.15 & 0.20 & 0.11 & Starker Rückgang \\
\hline Japan & 0.30 & 0.27 & 0.29 & 0.28 & 0.20 & 0.22 & Rückgang \\
\hline Luxemburg & 0.26 & 0.35 & 0.40 & 0.36 & 0.44 & 0.50 & Steigerung \\
\hline Niederlande & 0.86 & 0.82 & 0.76 & 0.81 & 0.81 & 0.81 & Rückgang \\
\hline Neuseeland & 0.26 & 0.25 & 0.24 & 0.23 & 0.21 & 0.25 & Schwankend \\
\hline Norwegen & 1.16 & 1.01 & 1.05 & 0.87 & 0.85 & 0.86 & Rückgang \\
\hline Portugal & 0.36 & 0.28 & 0.34 & 0.25 & 0.21 & 0.25 & Rückgang \\
\hline Spanien & 0.27 & 0.28 & 0.28 & 0.24 & 0.22 & 0.23 & Rückgang \\
\hline Schweden & 1.03 & 0.99 & 0.96 & 0.77 & 0.84 & 0.76 & Rückgang \\
\hline Schweiz & 0.39 & 0.34 & 0.36 & 0.34 & 0.34 & 0.32 & Rückgang** \\
\hline Grossbritannien & 0.31 & 0.31 & 0.31 & 0.29 & 0.27 & 0.26 & Rückgang \\
\hline Vereinigte Staaten & 0.20 & 0.15 & 0.14 & 0.10 & 0.12 & 0.08 & Stark.Rückgang *** \\
\hline Gewogener Mittelwert & & & & & & & \\
der DAC-Länder & $\mathbf{0 . 3 3}$ & $\mathbf{0 . 3 0}$ & $\mathbf{0 . 3 0}$ & $\mathbf{0 . 2 7}$ & $\mathbf{0 . 2 5}$ & $\mathbf{0 . 2 2}$ & \\
\hline In Milliarden \$ & 60.85 & 56.49 & 59.15 & 58.93 & 55.49 & 47.58 & \\
\hline
\end{tabular}

Quellen: Rapport du CAD 1997, "Coopération pour le développement. Efforts et politiques des membres du Comité d'aide au développement", OCDE, Paris, 1998 (für die Angaben von 1992 bis 1996); Pressemitteilung der OECD, Paris, 18. Juni 1998 (für die provisorischen Angaben für 1997).

* Der Rückgang von 1996 auf 1997 ist durch eine Änderung des Systems der volkswirtschaftlichen Gesamtrechnung bedingt, die eine Höherbewertung des BSP und dadurch eine Senkung des Quotienten öffentliche EZA zum BSP zur Folge hatte.

** Die öffentliche Entwicklungshilfe der Schweiz war 1992 wegen des Beitritts der Eidgenossenschaft zu den Bretton-Woods-Institutionen besonders hoch.

*** Zwei Drittel des Rückgangs der öffentlichen Entwicklungshilfe der Vereinigten Staaten zwischen 1996 und 1997 lassen sich durch die Streichung Israels von der Liste der Entwicklungsländer erklären, da Israel das Hauptempfängerland der amerikanischen Hilfe war.

Tabelle 18 zeigt die Entwicklung der Leistungen der DAC-Länder seit 1992, in Prozent des BSP. Dabei lassen sich mehrere Aspekte hervorheben:

๖ Seit 1992 haben alle Länder - mit Ausnahme von Irland, Luxemburg, Neuseeland und Dänemark - einen Rückgang ihrer öffentlichen Entwicklungshilfe verzeichnet. Drei Länder - Italien, die Vereinigten Staaten und Finnland - haben ihre Hilfe sehr stark gekürzt. Bei Österreich, den Niederlanden, Spanien und Grossbritannien fiel der Rückgang etwas mässiger aus. 
口 Die Kürzungen der Entwicklungshilfebudgets in den Ländern der G-7 erklären praktisch den gesamten seit einiger Zeit verzeichneten Rückgang der öffentlichen Entwicklungszusammenarbeit aller DAC-Länder. Von den Ländern der G-7 haben lediglich Kanada und Japan ihre öffentliche Hilfe im Realwert erhöht. Die öffentliche Hilfe der anderen, nicht der G-7 angehörenden Länder hat sich in den letzten Jahren relativ wenig verändert.

a Die Hilfeleistungen einiger Länder sind von einem Jahr zum anderen, vor allem wegen der unregelmässigen Auszahlung der multilateralen Beiträge, ungleichmässiger geworden. Der starke Rückgang der Beiträge an die multilateralen Organisationen erklärt zum Beispiel den scharfen Abfall der Hilfe Japans und Frankreichs zwischen 1995 und 1996 und das besonders niedrige Niveau der öffentlichen Hilfe Kanadas, der USA und Italiens im Jahr 1995.

a Die Entwicklungshilfe einiger kleiner Länder (Irland, Luxemburg) hat sich seit 1992 beträchtlich erhöht.

• Einer der Hauptgründe für den Rückgang der öffentlichen Entwicklungszusammenarbeit war - besonders in den europäischen Ländern - der Wille, die Haushaltsdefizite in mehreren Ländern zu senken, um den Kriterien des Maastricht-Vertrags zu entsprechen. Die Länder mit den höchsten Haushaltsdefiziten haben die Entwicklungshilfebudgets am schnellsten gekürzt.

Die Finanzierung durch die Entwicklungshilfe bleibt indes bei einigen Ländern weiterhin hoch, auch wenn die privaten Leistungen (Direktinvestitionen und Portfolioanlagen) in den letzten Jahren stark zugenommen haben (Rückgang 1997 infolge der Asienkrise). Die Privatkapitalbeiträge sind von 77,3 Milliarden Dollar 1992 auf 286,3 Milliarden Dollar 1996 angestiegen. Diese Beiträge konzentrieren sich aber auf eine sehr geringe Anzahl von Ländern, wogegen andere Gebiete ausserhalb dieser Kapitalströme bleiben und folglich weiterhin von Finanzmitteln wie der öffentlichen Entwicklungshilfe sehr abhängig sind. Zum Beispiel haben die afrikanischen Länder südlich der Sahara pro Einwohner durchschnittlich 27 Dollar an Hilfe und 3 Dollar an ausländischen Direktinvestitionen erhalten ${ }^{1}$. Die wichtigsten Bestimmungsländer der Privatkapitalbeiträge sind weiterhin die dynamischsten Volkswirtschaften Asiens, Europas und Lateinamerikas.

\section{$\square$ Hilfe für die am wenigsten entwickelten Länder (LDC)}

Die Hilfe für die am wenigsten entwickelten Länder bleibt weit hinter den Zielsetzungen der internationalen Gemeinschaft zurück. Die Geberländer hatten sich zum Ziel gesetzt, für diese Ländergruppe Hilfeleistungen von mindestens 0,15\% des BSP aufzubringen. Jedoch lag der Durchschnittswert der DAC-Länder 1996 lediglich bei 0,06\% des BSP. Dänemark, Norwegen, Schweden und die Niederlande überschreiten das gesteckte Ziel der Hilfe für die am wenigsten entwickelten Länder erheblich (mit jeweils 33\% für die beiden ersteren Länder und 0,24\% bzw. 0,23\% für die beiden letzteren). Unter dem Durchschnittswert des DAC liegen die Vereinigten Staaten (mit nur 0,02\% der Hilfe für die LDC), Japan, Österreich und Neuseeland. Die Schweiz brachte 1996 0,10\% ihrer Hilfeleistungen für die am wenigsten entwickelten Länder auf². 

in der Schweiz

Die Schweiz hat sich dem UN-Ziel einer Entwicklungshilfe von 0,7\% des BSP niemals angeschlossen. Anfang der 90er Jahre, insbesondere auf der Konferenz von Rio über Umwelt und Entwicklung, hatte der Bundesrat mehrmals seine Absicht erklärt, allmählich eine öffentliche Hilfe von $0,4 \%$ des BSP zu erreichen. In Anbetracht des Zustands der Bundesfinanzen und der vom Parlament verabschiedeten Budgetkürzungen entfernt sich die Schweiz jedoch von diesem Ziel, da die Hilfe in den letzten Jahren von 0,36\% des BSP 1994 auf 0,32\% 1997 zurückgegangen ist und die Aussichten für die kommenden Jahre weiterhin düster sind. Gemäss den Budgetkürzungen, die noch vom Parlament beschlossen werden können, könnte die öffentliche Entwicklungshilfe der Schweiz bis Ende des Jahrhunderts auf 0,30\% oder 0,29\% des BSP absinken.

Die öffentliche Entwicklungszusammenarbeit der Schweiz belief sich 1997 auf 1217 Millionen Franken (wovon 1193,8 Millionen vom Bund und 23,2 Millionen von den Kantonen und Gemeinden finanziert wurden). Die öffentliche Hilfe macht 3\% der Gesamtaufwendungen des Bundes aus.

\subsection{ORGANISATION UND VERWALTUNG DER HILFE DER SCHWEIZ}

\section{$\square$ Verwaltung der Hilfe im Rahmen der Bundesverwaltung}

Zwei Bundesämter teilen sich die Verwaltung des grössten Teils der öffentlichen Entwicklungszusammenarbeit. Die Direktion für Entwicklung und Zusammenarbeit (DEZA) im eidgenössischen Departement für auswärtige Angelegenheiten (EDA) hat 1997 rund 82\% der öffentlichen Hilfe (die technische Zusammenarbeit, einen Teil der Finanzhilfe, die humanitäre Hilfe) verwaltet. Das Bundesamt für Aussenwirtschaft (BAWI) im eidgenössischen Wirtschaftsdepartement hat 1997 10\% der öffentlichen Hilfe (wirtschafts- und handelspolitische Massnahmen) verwaltet. Das BAWI verwaltet ausserdem den grössten Teil der öffentlichen Hilfe für die mittel- und osteuropäischen Länder. Vertreter und Vertreterinnen von BAWI und DEZA gehören den meisten Delegationen bei internationalen Konferenzen an. Andere Departemente und Bundesämter sind in bestimmten Bereichen der internationalen Zusammenarbeit mit einbezogen: das Bundesamt für Gesundheitswesen für die WHO, das eidgenössische Finanzdepartement für den IWF, das Bundesamt für Umwelt, Wald und Landschaft für die internationalen Organisationen und Übereinkommen im Umweltbereich, das Bundesamt für Bildung und Wissenschaft bezüglich der Stipendien für Studienzwecke usw.

Der Bundesrat hat im Mai 1998 beschlossen, das BAWI und das Bundesamt für Wirtschaft und Arbeit (BWA, früher BIGA) zusammenzulegen, um alle Kompetenzen im wirtschaftlichen Bereich unter einem Dach zu vereinen (das neue Amt wird seinen Betrieb bis zum Jahr 2000 aufnehmen). Die Aspekte der Innen- und Aussenwirtschaftspolitik werden dadurch integriert. Der von Bundesrat Couchepin Ende November 1998 ernannte neue Staatssekretär David Syz, Nachfolger von Franz Blankart, soll die Zusammenlegung zwischen den beiden Bundesämtern durchführen und das neue Bundeswirtschaftsamt leiten. 
Die schweizerische Politik für Entwicklung und Zusammenarbeit beruht auf drei Säulen:

口 Dem Bundesgesetz über internationale Entwicklungszusammenarbeit und humanitäre Hilfe (vom 19. März 1976) und seiner Anwendungsverordnung (12. Dezember 1977). Das Gesetz legt fest, dass die Hilfe prioritär die Anstrengungen der ärmeren Entwicklungsländer, Regionen und Bevölkerungsgruppen fördern muss.

- Den mehrjährigen Rahmenkrediten, die den eidgenössischen Räten in «Botschaften des Bundesrates» unterbreitet werden. Der Nationalrat und der Ständerat müssen für jedes Kooperationsinstrument mehrjährige Finanzverpflichtungen (höchstens alle vier Jahre) bewilligen. Diese Unterlagen erlauben es, eine Bilanz der Erfahrungen der Vergangenheit zu ziehen und die Ausrichtungen der Hilfe für die kommenden Jahre festzulegen. Das Parlament setzt anschliessend die jährlichen Beträge der Hilfe im Rahmen der Verabschiedung des Jahresbudgets des Bundes fest. Tabelle 19 führt die Liste der wichtigsten zur Zeit in Kraft stehenden Rahmenkredite auf.

- Den Dokumenten, in denen die Hauptorientierungen der Entwicklungszusammenarbeit festgelegt sind. Das (1994 verabschiedete) «Leitbild Nord-Süd» stellt vier Ziele für die Entwicklungspolitik auf: 1) Wahrung und Förderung von Frieden und Sicherheit, Förderung von Menschenrechten, Demokratie und Rechtsstaat; 2) Förderung der Wohlfahrt; 3) Erhöhung der sozialen Gerechtigkeit; 4) Schutz der natürlichen Lebensgrundlagen. Das Dokument unterstreicht besonders die Notwendigkeit einer besseren Kohärenz zwischen der Politik der Entwicklungszusammenarbeit und den anderen Bereichen der Politik und der Aussenbeziehungen der Schweiz mit den Entwicklungsländern. Die zugrunde liegende Idee ist, die Widersprüche, die zwischen der Entwicklungszusammenarbeit und anderen Politiken (Agrarpolitik, Migrations-, Umwelt-, Exportförderungspolitik) bestehen können, besser zu ermitteln. Auf dieser Grundlage können die DEZA und das BAWI ihre Meinung zu Fragen abgeben, die streng genommen nicht die Entwicklungszusammenarbeit betreffen, aber Auswirkungen darauf haben können, zum Beispiel Waffenexporte, Bekämpfung von Korruption und Kapitalflucht, Zugang zum schweizerischen Markt für Produkte der Entwicklungsländer, Ausarbeitung eines Modells nachhaltiger Entwicklung für die Schweiz usw.

Der Bundesrat hat im Dezember 1998 seine neue Botschaft über die Weiterführung der technischen Zusammenarbeit und Finanzhilfe mit einem Rahmenkredit von 4 Milliarden Schweizer Franken für die Jahre 1999-2002 unterbreitet. Die Botschaft bietet nicht nur den Anlass, den finanziellen Rahmen festzulegen, sondern auch die Bilanz der vergangenen Aktivitäten zu ziehen und die grundlegenden Orientierungen der Hilfe für die kommenden Jahre aufzuzeigen (das Jahrbuch 2000 wird ausführlich auf den Inhalt der neuen Botschaft eingehen). 
Tabelle Nr. 19: Letzte bewilligte Rahmenkredite

\begin{tabular}{|c|c|c|c|}
\hline $\begin{array}{l}\text { Datum der } \\
\text { Botschaft } \\
\text { (Botschaftsnummer) } \\
\end{array}$ & Rahmenkredit & $\begin{array}{l}\text { Betrag } \\
\text { in Millionen } \\
\text { Franken }\end{array}$ & $\begin{array}{l}\text { Mindestlaufzeit } \\
\text { und } \\
\text { Inkrafttreten }\end{array}$ \\
\hline $\begin{array}{l}21.2 .1990 \\
(90.015)\end{array}$ & $\begin{array}{l}\text { Wirtschafts- und handels- } \\
\text { politische Massnahmen }\end{array}$ & 840 & $\begin{array}{l}4 \text { Jahre } \\
1991-1996\end{array}$ \\
\hline $\begin{array}{l}30.1 .1991 \\
(90.080)\end{array}$ & $\begin{array}{l}\text { Finanzierung von Entschuldungs- } \\
\text { massnahmen für Entwicklungs- } \\
\text { länder und Umweltprogramme }\end{array}$ & 700 & ab 1991 \\
\hline 15.5 .1991 & $\begin{array}{l}\text { Beitritt der Schweiz zu den } \\
\text { Bretton-Woods-Institutionen }\end{array}$ & 4986 & ab 17.5.1992 \\
\hline $\begin{array}{l}20.4 .1994 \\
(94.029)\end{array}$ & $\begin{array}{l}\text { Technische Zusammenarbeit } \\
\text { und Finanzhilfe }\end{array}$ & 3800 & $\begin{array}{l}4 \text { Jahre } \\
\text { ab 1.6.1995 }\end{array}$ \\
\hline $\begin{array}{l}31.5 .1995 \\
(95.041)\end{array}$ & $\begin{array}{l}\text { Beteiligung am Kapital } \\
\text { der regionalen Entwicklungsbanken }\end{array}$ & 800 & ab 1.1.1996 \\
\hline $\begin{array}{l}13.12 .1996 \\
(96.044)\end{array}$ & $\begin{array}{l}\text { Neuausrichtung von wirtschafts- } \\
\text { und handelspolitischen Massnahmen }\end{array}$ & 960 & $\begin{array}{l}4 \text { Jahre } \\
\text { ab 1.1.1997 }\end{array}$ \\
\hline $\begin{array}{l}20.11 .1996 \\
(96.092)\end{array}$ & Internationale humanitäre Hilfe & 1050 & $\begin{array}{l}4 \text { Jahre } \\
\text { ab 1.2.1998 }\end{array}$ \\
\hline 7.12.1998 & $\begin{array}{l}\text { Technische Zusammenarbeit } \\
\text { und Finanzhilfe }\end{array}$ & 4000 & $\begin{array}{l}4 \text { Jahre } \\
1999-2002\end{array}$ \\
\hline
\end{tabular}

Quellen: DEZA, BAWI.

\section{$\square$ Bericht über zehn Jahre internationaler Zusammenarbeit}

In Erfüllung des Postulats von Nationalrätin Rosmarie Zapfl-Helbling vom September 1996 hat der Bundesrat 1998 einen Bericht über die internationale Zusammenarbeit der Schweiz mit Ländern des Südens und des Ostens für die Jahre 1986-1995 veröffentlicht. Der Bericht weist auf die Veränderungen in der Weltpolitik und die neuen Herausforderungen für die internationale Zusammenarbeit hin. Er zieht auch eine Bilanz der Anpassungen der schweizerischen Entwicklungszusammenarbeit an diese Veränderungen und der Beiträge, die sie zur Lösung gewisser weltweiter Probleme zu leisten versucht.

Unter den wesentlichen globalen Veränderungen der Periode 1986-1995 wird im Bericht die Differenzierung der Situationen in der Welt hevorgehoben: Lage der neuen Industrieländer, Probleme der am wenigsten entwickelten Länder (LDC) und vor allem der wirtschaftliche und soziale Übergangsprozess im früheren Ostblock. Wenn soziale Gerechtigkeit und Armut auch weiterhin ein grundlegendes Problem bleiben, so wurde den Fragen des Schutzes der natürlichen Lebensgrundlagen und der Entwicklung demokratischerer Gesellschaften jedoch grössere Aufmerksamkeit geschenkt. Regierungen und öffentliche Meinung sind sich immer klarer bewusst geworden, dass gewisse grenzüberschreitende Probleme (Klimaveränderungen, Migrationen, Weiterverbreitung von Waffen, internationale Kriminalität und Drogen) für ihr Land weit grössere Risiken als die Eventualität herkömmlicher militärischer Agressionen in sich bergen. Der Begriff der «globalen menschlichen Sicherheit» beruht auf der Interdependez zwischen den Ländern und auf der Tatsache, dass Industriestaaten und Entwicklungsländer ein gemeinsames lebenswichtiges Interesse daran haben, gewisse weltweite Probleme zu lösen.

Mehrere Aspekte nehmen im Rahmen der internationalen Zusammenarbeit an Bedeutung zu: Berücksichtigung der Notwendigkeit einer guten Regierungs- 
führung ${ }^{3}$, Demokratisierung und eine offenere politische Partizipation an der Zivilgesellschaft, Berücksichtigung der Stellung der Frauen, Umwelt und Entwicklung.

Der Bericht führt eine Reihe von Instrumenten der Zusammenarbeit auf, die von der Schweiz erstellt wurden, um gewissen Herausforderungen zu begegnen. Einige Ansätze der schweizerischen Entwicklungszusammenarbeit sind originell und innovativ: Anliegen der Kohärenz zwischen der Politik der Entwicklungszusammenarbeit und den anderen Politiken der Eidgenossenschaft gegenüber den Entwicklungsländern, Entschuldungspolitik (Gegenwertfonds, Initiativen für die am meisten verschuldeten Länder). Die schweizerische Entwicklungszusammenarbeit misst ferner der partizipativen Entwicklung (Selbsthilfe, Förderung von Basisorganisationen) sowie der Zusammenarbeit mit den privaten Hilfswerken und den Kreisen der schweizerischen Privatwirtschaft grosse Bedeutung bei.

In den allgemeinen Schlussfolgerungen über die internationale Zusammenarbeit bekräftigt der Bericht, dass die Zusammenarbeit eine notwendige Investition in unser aller Zukunft ist und dass die Bestrebungen für eine qualitative Verbesserung der Hilfe fortgesetzt werden müssen. Der Bericht stellt fest, die schweizerische Zusammenarbeit habe bewiesen, dass sie gegenüber den neuen Herausforderungen dynamisch und flexibel bleiben konnte. Er hebt die Komplementarität zwischen multi- und bilateraler Zusammenarbeit hervor. Er betont erneut die Notwendigkeit einer besseren Kohärenz in den Beziehungen mit den Entwicklungsländern und erklärt, die menschliche Entwicklung müsse das Hauptanliegen der schweizerischen Zusammenarbeit bleiben.

\subsection{ALLGEMEINE ENTWICKLUNG DER BILATERALEN HILFE}

Die bilaterale Hilfe ist seit 1994 zurückgegangen, wobei sie von 985 Millionen Franken 1994 auf 895 Millionen Franken 1996 und 841 Millionen Franken 1997 gesunken ist. Arbeitsgrundlagen für die Ausrichtung der bilateralen Zusammenarbeit der DEZA sind die nach Themen oder Sektoren angelegten Strategiepapiere (zum Beispiel Politik der Förderung von Handwerk und Industrie, städtische Entwicklung), die Länderprogramme (von DEZA und BAWI erstellte Fünfjahresprogramme) und die Jahresprogramme.

Das für jedes Schwerpunktland erstellte Länderprogramm informiert über die Situation und die Bedürfnisse des betreffenden Staates. Es legt die allgemeinen Ziele der schweizerischen Zusammenarbeit, die vorgesehenen Strategien und die zur Erreichung der Ziele erforderlichen menschlichen und finanziellen Ressourcen fest. Die Jahresprogramme geben die Durchführungsphasen an und ermöglichen eine Anpassung der Länderprogramme. In den Länderprogrammen wird heute eine bessere Artikulation zwischen den verschiedenen Interventionsebenen - lokale Zielbevölkerung (Mikroebene), institutioneller Rahmen (Mesoebene) und politische Unterstïtzung (Makroebene) - angestrebt.

3. Der Begriff «Good Governance» oder «gute Regierungsführung» umfasst folgende Aspekte: Förderung der Rechtsstaatlichkeit, effiziente Verwaltung, Bekämpfung der Korruption und Verringerung übermässiger Militärausgaben, 
Die Koordinationsbüros sichern eine dezentralisierte Präsenz der DEZA bei den Partnern in den sogenannten Schwerpunktländern. Sie spielen eine wesentliche Rolle, da sie den lokalen Partnern und den administrativen Realitäten der Interventionsländer der schweizerischen Hilfe nahestehen.

Die Zusammenarbeit der Schweiz fördert die lokale Aneignung der Projekte und Programme (Berücksichtigung der von der Bevölkerung, der die Hilfe zugute kommt, ausgedrückten Bedürfnisse, Zusammenarbeit mit den örtlichen Organisationen und der Zivilgesellschaft, Selbsthilfe, Förderung von Basisorganisationen).

\section{$\square$ Schwerpunktländer der schweizerischen Hilfe}

Die DEZA verfolgt eine Politik der Konzentration der Hilfe auf bestimmte Länder. Die Anzahl der sogenannten Schwerpunktländer ist allmählich von 20 auf 16 zurückgegangen. Die anderen Bereiche der Entwicklungszusammenarbeit folgen nicht dem Kriterium der Konzentration auf die gleichen Länder wie die technische Zusammenarbeit der DEZA. Die Auszahlung von Stipendien für Studienzwecke, die humanitäre Hilfe, die vom BAWI finanzierten Massnahmen und die Beiträge an die Nichtregierungsorganisationen sind nicht zwangsläufig vor allem auf die sogenannten Schwerpunktländer gerichtet. Das BAWI kann seine Tätigkeit aufgrund der Art seiner Instrumente (Entschuldungsmassnahmen, Zahlungsbilanzhilfe usw.) geographisch nicht in gleicher Weise wie die DEZA konzentrieren.

- Liste der Schwerpunktländer oder Schwerpunktregionen für die Hilfe der DEZA. Afrika: Benin, Burkina Faso, Madagaskar, Mali, Mosambik, Niger, Tansania und Tschad. Lateinamerika: Bolivien, Nicaragua/Mittelamerika und Peru. Asien: Bangladesch, Indien, Nepal, Pakistan und Vietnam/Mekonggebiet.

๖ Liste der wichtigsten Interventionsländer des BAWI, die auch Schwerpunktländer der DEZA sind: Bolivien, Burkina Faso, Indien, Mosambik, Nicaragua, Pakistan, Peru, Tansania und Vietnam.

口 Weitere Hauptinterventionsländer des BAWI (in denen die DEZA nur wenig vertreten ist): Ägypten, China, Indonesien (Schwerpunktland der DEZA bis 1995) und Uganda.

Tabelle 3.3.A. im Statistischen Teil führt die von der DEZA für technische Zusammenarbeit und Finanzhilfe in den Schwerpunktländern der DEZA aufgebrachten Beträge an. Die höchsten Auszahlungen entfielen auf Indien (1997: 28,1 Millionen Franken), Mittelamerika (21,5 Millionen Franken) und Tansania (20,5 Millionen). Seit 1997 ist Vietnam auf der Liste der Schwerpunktländer an die Stelle Indiens getreten. Die Zusammenarbeit mit Madagaskar ist 1998, namentlich infolge der Langsamkeit bei der Untersuchung über die Ermordung eines Schweizer Entwicklungshelfers zwei Jahre zuvor, unterbrochen worden.

\section{$\square$ Geographische Aufteilung der bilateralen Hilfe}

Graphik 5 führt die Hauptempfängerländer der schweizerischen bilateralen Hilfe auf und stellt die Unterschiede nach der Art der Hilfe heraus. Einige Länder (Ex-Jugoslawien und Ruanda) erhalten viel humanitäre Hilfe. Andere Länder sind wichtige Partner für die Entwicklungszusammenarbeit, während die wirtschafts- und handelspolitischen Massnahmen einen Grossteil der Hilfe für gewisse Länder (Bolivien, Burkina Faso, Mosambik und Tansania) ausmachen. 
Graphik Nr. 5: Wichtigste Empfängerländer und -regionen der schweizerischen Entwicklungszusammenarbeit und Aufteilung nach Art der Hilfe Kumulierter Gesamtbetrag der bilateralen Hilfe, in Millionen Franken
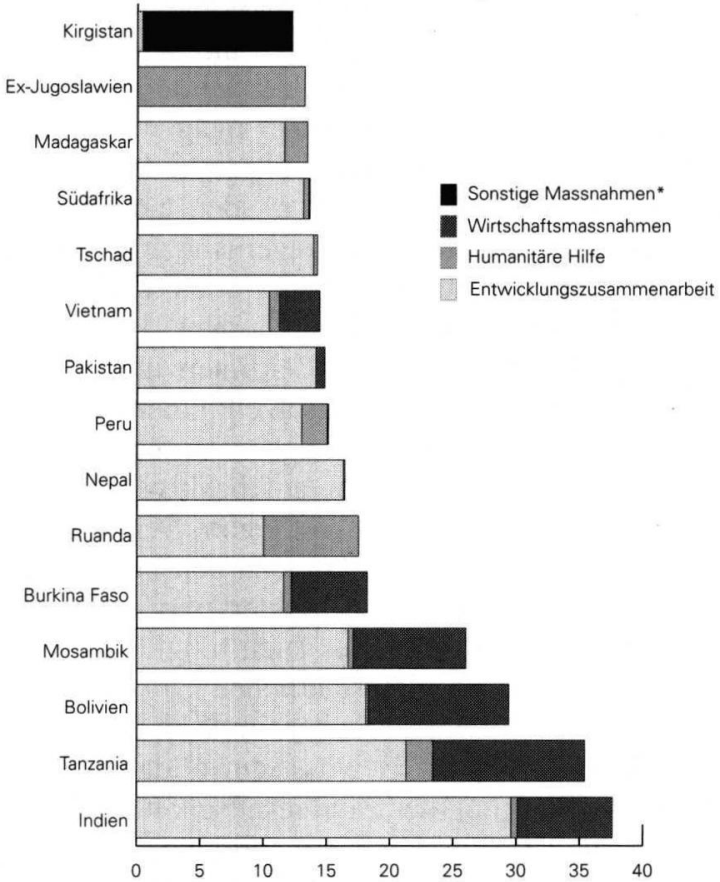

Quelle: Anhand von Tabelle 3 des Jahresberichts 1997, Entwicklungszusammenarbeit, DEZA, BAWI, 1998, erstellte Graphik.

* Die sonstigen Massnahmen umfassen beispielsweise die Finanzhilfe.

\section{$\square$ Sektorielle Aufteilung der Hilfe und Schwerpunktthemen}

Die Schwerpunktbereiche der bilateralen Hilfe sind Landwirtschaft und Viehzucht (16\% der bilateralen Hilfe der DEZA 1997), Infrastruktur, Wasser und Energie (14\% der Hilfe), Erziehung, Information und Kultur (13\%), Wald und Umwelt (9\%) sowie Gesundheit, Ernährung und Bevölkerung (8\%). Mehreren Themen kommt bei der Verwaltung der Projekte der Entwicklungszusammenarbeit besondere Beachtung zu: nachhaltige Entwicklung, ausgewogene Entwicklung von Mann und Frau (Gender), Partizipation der von der Hilfe betroffenen Bevölkerung. Statt sich darauf zu beschränken, spezifische Projekte zu einem dieser Bereiche zu erstellen, sollten diese Themen in allen Hilfsprogrammen berücksichtigt werden. Die Sektorpolitiken werden von der DEZA regelmässig überarbeitet. Das BAWI konzentriert sich auf die Bereiche, in denen die schweizerische Wirtschaft einen komparativen Vorteil aufweist, wie auch auf Wirtschaftsreformen, Technologietransfer (besonders für den Umweltschutz) und auf die Mobilisierung privater Ressourcen.

Die DEZA hat 1998 ein Bestandsverzeichnis der Aktivitäten im städtischen Bereich aufgestellt. Aus dem Bericht geht hervor, dass von 1993 bis 1998 über 270 Aktivitäten im städtischen Bereich für Projekte durchgeführt wurden, die einem Drittel des bilateralen Jahresbudgets der DEZA entsprechen. 


\section{$\square$ Förderung der Menschenrechte}

Die Leitlinien über «Förderung der Menschenrechte und Entwicklungszusammenarbeit» wurden von DEZA und BAWI im Februar 1997 erarbeitet $^{4}$. Die Unterlage weist auf die grossen Linien der Aussenpolitik des Bundes im Bereich der Menschenrechte sowie auf die Auswirkungen dieser Politik bei der Durchführung von Projekten der Entwicklungszusammenarbeit hin. Das Engagement zugunsten der Menschenrechte gehört zu den fünf vorrangigen Zielen, die im Bericht des Bundesrates über die Aussenpolitik der Schweiz in den 90er Jahren festgelegt wurden. Das Leitbild Nord-Süd bekräftigt dieses Engagement und setzt es als eines der strategischen Ziele der Aktion zur Unterstützung der Entwicklungsländer fest (Wahrung und Förderung von Frieden und Sicherheit, Förderung von Menschenrechten, Demokratie und Rechtsstaat). Die Leitlinien über die Förderung der Menschenrechte stellen eine Reihe von Grundsätzen in den Vordergrund, die bei der Durchführung von Projekten der Entwicklungszusammenarbeit einzuhalten sind. Der Bund will das Schwergewicht mehr auf positive Massnahmen und auf den Dialog als auf eine negative Konditionalität legen. Der Abbruch von Kooperationsprojekten oder die Einstellung der Zusammenarbeit mit einem Land stellt eine letztmögliche Lösung im Fall schwerwiegender und systematischer Menschenrechtsverletzungen dar. In diesem Fall ist es auch möglich, den staatlichen Trägern die Hilfe zu entziehen und sie nichtstaatlichen Organisationen zu übertragen. Die Praxis der DEZA wird de facto eher im Sinne von Anreizmassnahmen gehandhabt, mit Massnahmen zur Bewusstseinsbildung der Bevölkerung, Unterstützung der Ärmsten, Stärkung der Zivilgesellschaft, Verbesserung von Gesetzgebung, Rechtsvollzug und Rechtsschutz, Ausbildung, Förderung der Rechtsstaatlichkeit und Unterstützung unabhängiger Medien. Im Dokument wird anerkannt, dass sich eine glaubwürdige Menschenrechtspolitik auch in einer besseren Kohärenz zwischen den verschiedenen Instrumenten der schweizerischen Aussenpolitik niederschlagen sollte. Die Kohärenz soll zwischen der Politik der Entwicklungszusammenarbeit und den anderen Politikbereichen angestrebt werden, zum Beispiel: Migrationspolitik und Rückkehrhilfe, Politik der Export- und Investitionsförderung, Exportbewilligungen für Kriegsmaterial. Für das BAWI ist es auch möglich, sich zugunsten der Menschenrechte einzusetzen durch eine Politik der Liberalisierung der Wirtschaft der Entwicklungsländer, der Öffnung gegenüber der Aussenwelt und der Integration in die Weltwirtschaft, indem man dadurch die vollständige Isolierung eines Landes vermeidet.

\subsection{BILATERALE HILFE - WIRTSCHAFTS- UND HANDELSPOLITISCHE MASSNAHMEN}

Diese Instrumente der Zusammenarbeit werden vom Bundesamt für Aussenwirtschaft (BAWI) verwaltet. Der fünfte Rahmenkredit über die Weiterführung und Neuausrichtung von wirtschafts- und handelspolitischen Massnahmen ist Anfang 1997 in Kraft getreten. Das BAWI schlug eine Neuorientierung der Massnahmen vor, wobei der Schwerpunkt viel stärker auf die Zusammenarbeit mit dem Privatsektor gelegt wurde. Die privaten Unternehmen führen nicht

4. Die Leitlinien sind in der DEZA-Unterlage «Förderung der Menschenrechte und Entwicklungszusammenarbeit», Bern, DEZA, 1997 (24 Seiten) veröffentlicht. Zum Thema «Menschenrechte und internationale Zusammenarbeit», siehe auch das DEZA-Magazin «Eine Welt», Nr. 4, Dezember 1998. 
mehr nur Aufträge aus, sondern die Kooperationsprojekte werden zunehmend in direkter Zusammenarbeit mit den Privatfirmen entwickelt und konzipiert. Die Entwicklungsländer werden bei ihren Bemühungen gefördert, sich in die Weltwirtschaft einzugliedern und Investitionen anzuziehen.

JSDW 1997, Die Botschaft wurde ausführlich behandelt, S.157-170.

Die nachstehende Tabelle 20 gibt einen Überblick über die Aufwendungen des BAWI für die verschiedenen wirtschafts- und handelspolitischen Massnahmen von 1991 bis 1998. Die den einzelnen Instrumente zugemessene Bedeutung hat sich in den letzten acht Jahren geändert. Die Fördermassnahmen im Rohstoffbereich sind seit 1991 stark zurückgegangen. Die Massnahmen zur Förderung der Investitionen des Privatsektors in den Entwicklungsländern gewinnen an Bedeutung, während die Massnahmen zur Stärkung der Rahmenbedingungen in den Entwicklungsländern weiterhin ein wichtiger Pfeiler der schweizerischen wirtschaftlichen Zusammenarbeit bleiben.

Die vom BAWI verwaltete Finanzhilfe belief sich 1997 auf 104 Millionen Franken und 1998 auf 152 Millionen Franken. Die Mischfinanzierungen machten 1998 nur noch 15\% des Gesamtvolumens aus, wogegen sie von 1990 bis 1994 zwischen ein Drittel und nahezu der Hälfte des Gesamtvolumens der Wirtschaftshilfe ausmachten. Die Investitionsförderungsmassnahmen nehmen in den letzten Jahren an Bedeutung zu und erreichten 1998 40\% der Hilfe.

Tabelle Nr. 20: Wirtschafts- und handelspolitische Massnahmen Aufwendungen nach Instrumenten, von 1990 bis 1998

\begin{tabular}{|c|c|c|c|c|c|c|c|c|c|c|}
\hline \multirow{3}{*}{$\begin{array}{l}\text { Art } \\
\text { der } \\
\text { Massnahmen }\end{array}$} & \multicolumn{10}{|c|}{ Aufwendungen } \\
\hline & in Mio. & in & \multicolumn{6}{|c|}{ in Millionen Franken } & \multirow{2}{*}{\multicolumn{2}{|c|}{$\begin{array}{c}\text { in Mio. ir } \\
\text { Fr. } \\
1998\end{array}$}} \\
\hline & \multicolumn{2}{|c|}{1991} & \multirow{2}{*}{$\begin{array}{r}1992 \\
43.5\end{array}$} & \multirow{2}{*}{$\begin{array}{r}1993 \\
25.5\end{array}$} & \multirow{2}{*}{$\begin{array}{r}1994 \\
29.2\end{array}$} & \multirow{2}{*}{$\begin{array}{r}1995 \\
13.0\end{array}$} & \multirow{2}{*}{$\begin{array}{r}1996 \\
29.5\end{array}$} & \multirow{2}{*}{$\begin{array}{r}1997 \\
40.2\end{array}$} & & \\
\hline Zahlungsbilanzhilfe & 52.2 & 22.9 & & & & & & & 10.5 & 6.9 \\
\hline $\begin{array}{l}\text { Entschuldungs- } \\
\text { massnahmen }\end{array}$ & 80.0 & 35.1 & 58.9 & 48.9 & 41.4 & 68.4 & 38.1 & 21.2 & 40.4 & 26.6 \\
\hline Handelsförderung & 5.8 & 2.5 & 5.2 & 5.3 & 5.1 & 7.5 & 13.5 & 4.8 & 10.2 & 6.7 \\
\hline Rohstoffe & 16.9 & 7.4 & 11.8 & 8.1 & 3.5 & 7.3 & 3.3 & 3.6 & 3.4 & 2.2 \\
\hline Investitionsförderung & 4.1 & 1.8 & 3.1 & 2.9 & 23.0 & 9.3 & 21.0 & 20.0 & 61.0 & 40.2 \\
\hline Mischfinanzierungen & 67.7 & 29.6 & 83.3 & 87.4 & 75.4 & 26.9 & 27.7 & 10.0 & 22.1 & 14.6 \\
\hline Studien und Verwaltung & 1.5 & 0.7 & 2.3 & 2.8 & 2.5 & 3.4 & 2.6 & 4.1 & 4.8 & 3.2 \\
\hline $\begin{array}{l}\text { Gesamtbetrag der } \\
\text { Wirtschaftsmassnahmen }\end{array}$ & 228.3 & 100.0 & 208.2 & 180.9 & 180.2 & 135.9 & 135.7 & 103.7 & 151.7 & 100.0 \\
\hline
\end{tabular}

Quelle: Nach Angaben des BAWI erstellte Tabelle.

\section{$\square$ Zahlungsbilanzhilfe}

Dieses Instrument der Entwicklungszusammenarbeit gestattet es in bestimmten Situationen, Einfuhren lebensnotwendiger Güter aus der Schweiz (medizinische Geräte, technisches Material) zu finanzieren, und im allgemeineren Fall die Regierung, der die Hilfe zugutekommt, beispielsweise bei der Durchführung einer Strukturanpassung finanziell zu unterstützen. 1997 hat die Schweiz zwei neue Zahlungsbilanzhilfeabkommen mit dem Senegal (13 Millionen Franken) und mit Tansania (12 Millionen Franken, vierte Zahlungsbilanzhilfe dieser Art) abgeschlossen, während Auszahlungen in Verbindung mit laufenden Aktionen in Bolivien, Burkina Faso, Eritrea und Mosambik erfolgten. 1998 wurde ein 
weiteres Abkommen mit Mosambik (einem Schwerpunktland der schweizerischen Entwicklungszusammenarbeit) über eine fünfte Zahlungsbilanzhilfe im Gesamtbetrag von 18 Millionen Franken abgeschlossen. Diese Aktion soll sich über zwei Jahre hinziehen und Mosambik in die Lage versetzen, seine Finanzsanierung zu konsolidieren und sein Programm wirtschaftlicher und struktureller Reformen fortzuführen. Die Auszahlungen des BAWI beliefen sich 1998 auf 10,5 Millionen Franken, davon 8,3 Millionen für Mosambik.

\section{$\square$ Entschuldungsmassnahmen}

Die Entschuldungsmassnahmen wurden 1991 anlässlich der Bewilligung des Rahmenkredits zum 700. Jahrestag der Eidgenossenschaft, nach der Petition der schweizerischen Hilfswerke zugunsten der Entschuldung eingeführt. Vom Gesamtbetrag des Rahmenkredits in Höhe von 700 Millionen Franken wurden 400 Millionen zur Unterstützung von Entschuldungsmassnahmen bereitgestellt. Das schweizerische Entschuldungsprogramm hat (beispielsweise mit den Gegenwertfonds) originelle Lösungen und neue Wege vorgeschlagen. Die Schweiz ist in den Bretton-Woods-Institutionen für den Rückkauf der multilateralen Schulden und für die Entschuldung der hochverschuldeten armen Länder eingetreten. Die Aufwendungen für Entschuldungsmassnahmen beliefen sich 1998 auf 40,4 Millionen Franken, davon 18 Millionen Franken für die von der HIPC-Initiative betroffenen Länder, 7,4 Millionen Franken für Nicaragua, 5 Millionen Franken für Guinea-Bissau (und 8,3 Millionen für andere afrikanische Länder).

$\square$ JSDW 1999, Kapitel 1 über die internationalen Finanzbeziehungen, Punkt 1.2. mit den multilateralen Entschuldungsmassnahmen und den Massnahmen der Schweiz und Punkt 1.4. zur Politik der Schweiz im Rahmen von IWF und Weltbank.

\section{$\square$ Investitionsförderung}

Der neue Rahmenkredit schlug eine Reihe neuer Instrumente der Entwicklungszusammenarbeit vor, die dazu bestimmt sind, die Ausfuhren und die Investitionen in die Entwicklungsländer zu beleben:

๖ Die neue Schweizerische Gesellschaft für Entwicklungsfinanzierung (SGE) soll eine Unterstützung der Unternehmen der OECD-Staaten (insbesondere der KMU) ermöglichen, welche Joint-Ventures einführen oder bestehende Unternehmen in einem Entwicklungsland oder einem osteuropäischen Land modernisieren wollen. Die Gesellschaft für Entwicklungsfinanzierung ist ein Gemeinschaftsunternehmen des schweizerischen privaten und öffentlichen Sektors. Das vorgesehene Kapital von 100 Millionen Franken wird zu 51\% von privaten Aktionären und zu 49\% vom Bund gezeichnet. Die neue SGE wird ab Frühjahr 1999 operationell sein. Die SGE gewährt ein Risikokapital für Direktinvestitionsprojekte und wird Dienstleistungen (Managementhilfe) erbringen. Die Beschlüsse erfolgen anhand der Rentabilität des Projekts und entwicklungspolitischer Kriterien.

口 Die übrigen im Rahmenkredit vorgesehenen Investitionsförderungsmassnahmen umfassen die Unterstützung zur Anbahnung von Geschäftsbeziehungen (zwischen Unternehmen des Nordens und des Südens, siehe nachstehend die Stiftung SOFI), die Finanzierung von Studien für Investitionen und Ausbildungstätigkeiten des Privatsektors sowie die Förderung des Transfers umweltverträglicher Technologien. 
Das Programm zur Anbahnung von Geschäftsbeziehungen zwischen Schweizer Firmen und Unternehmen der Entwicklungs- und Transitionsländer wird von der neuen Stiftung SOFI (Swiss Organisation for Facilitating Investments) verwaltet. Diese Stiftung wurde im Juli 1997 vom BAWI mit dem Ziel gegründet, die schweizerischen Investitionen in den Entwicklungsländern und Transitionsländern zu fördern. Die SOFI liefert Informationen über potentielle Märkte (Rahmenbedingungen in bestimmten Ländern, lokale Gesetzgebung, Investitionsanreizmassnahmen, Daten über Arbeitsmärkte, Besteuerung usw.). Die SOFI leistet Hilfe bei der Kontaktaufnahme zwischen Partnern (für Joint-Ventures oder andere Projekte langfristiger Zusammenarbeit): Suche nach potentiellen Partnern, Beratung, Durchführbarkeitsstudien, Begleitung und Vermittlung in der Verhandlungsphase, Unterstützung bei der Projektvorbereitung und umsetzung. Die Stiftung soll auf diese Weise erlauben, den Know-How-Transfer zwischen Schweizer Firmen (vor allem KMU) und Unternehmen der Partnerländer zu fördern ${ }^{5}$.

Der Bund hat ferner einen Risikokapitalfonds, den «Swiss Technology Venture Capital Fund» eingerichtet, welcher Gemeinschaftsunternehmen (Joint-Ventures) - hauptsächlich in Indien - finanzieren soll. Der Chinesisch-Schweizerische Partnerschaftsfonds wurde im Dezember 1997 gegründet, um die schweizerischen kleinen und mittleren Unternehmen zu unterstützen, die sich auf dem chinesischen Markt niederlassen wollen.

1998 entfielen die Hauptaufwendungen im Bereich der Investitionsförderung auf Indien (17,1 Millionen Franken), China (10,8 Millionen Franken) und auf die Insel Mauritius (10,5 Millionen Franken). Die Gesamtaufwendungen beliefen sich auf 61 Millionen Franken) (gegenüber 20 Millionen Franken 1997).

$\square$ JSDW 1999, Kapitel 7 «Aussenwirtschaftspolitik«, Handels- und Investitionsförderungspolitik.

\section{$\square$ Mischfinanzierungen}

Mischfinanzierungen setzen sich aus einem Geschenk des Bundes und einem von einem Schweizer Bankenkonsortium (zu Marktkonditionen) vergebenen Darlehen zusammen. Diese Kredite erlauben es dem Partnerland, Ausrüstungsgüter und Dienstleistungen in der Schweiz (für mindestens $50 \%$ des Kredits) oder in anderen Ländern zu beziehen. Acht Mischfinanzierungsabkommen haben 1997 und 1998 zu Auszahlungen geführt. Die Bedeutung dieses Instruments ist heute viel geringer als vor einigen Jahren, da die Aufwendungen für die laufenden Mischfinanzierungen 1997 lediglich 10 Millionen Franken (gegenüber 75 bis 87 Millionen Franken in den Jahren 1992-1994) erreichten. Der Hauptgrund für diesen Rückgang ist in der Schwierigkeit der Schweizer Unternehmen zu suchen, Projekte zu ermitteln, die den neuen OECD-Bestimmungen entsprechen. Die Verhaltensregeln der OECD beschränken den Einsatz von Mischfinanzierungen auf kommerziell nicht tragfähige Projekte. Die Mischfinanzierungen haben 1998 wiederum zu Auszahlungen von 22,1 Millionen Franken geführt, davon 8,8 Millionen für Ägypten, 4,4 Millionen für Jordanien, 3,6 Millionen für China, 1,6 Millionen für Tunesien und 1,3 Millionen für Vietnam.

Ein Abkommen über eine vierte Mischfinanzierung in Höhe von 80 Millionen Franken wurde Ende 1997 mit Ägypten abgeschlossen. 


\subsection{HUMANITÄRE HILFE}

Die humanitäre Hilfe kann im Rahmen der bilateralen oder multilateralen Hilfe erfolgen. 1997 haben die eidgenössischen Räte einen neuen Rahmenkredit von 1,05 Milliarden Franken für eine Laufzeit von mindestens vier Jahren gebilligt (vgl. Jahrbuch 1998, S. 327-330). Der Rahmenkredit über humanitäre Hilfe betrifft die Entwicklungsländer, aber auch die Länder Mittel- und Osteuropas (für letztere ist die Hilfe allerdings nicht in der öffentlichen Entwicklungshilfe, sondern in dem Aggregat «öffentliche Hilfe für die Transitionsländer» enthalten). Alljährlich werden die zur Verfügung gestellten Zahlungskredite von den eidgenössischen Räten im Rahmen des Bundesbudgets festgesetzt (156 Millionen Franken für 1997 und 158 Millionen Franken für 1998). Ein anderer Rahmenkredit regelt die ordentlichen Beiträge an das Sitzbudget des IKRK (vier Jahre lang 65 Millionen Franken). Ein neuer Rahmenkredit für das Sitzbudget des IKRK wurde vom Parlament im Dezember 1997 angenommen. Dieses Budget beläuft sich auf 275 Millionen Franken für die Jahre 1998 bis 2001.

Die humanitäre Hilfe des Bundes belief sich 1997 auf 222,1 Millionen Franken, wovon 217,1 Millionen in der öffentlichen Entwicklungshilfe verbucht waren (dies entspricht 17,8\% der gesamten öffentlichen Entwicklungshilfe). Die humanitäre Hilfe ist in den letzten Jahren zurückgegangen (230,4 Millionen Franken 1996, gegenüber 253,7 Millionen 1993). 1997 waren die Hauptempfängerländer der humanitären Hilfe Ex-Jugoslawien (13,2 Millionen Franken), Angola (8,3 Millionen), die Kaukasusrepubliken (8,2 Millionen), Ruanda (7,5) und Nordkorea $(6,3)$.

Von den 222 Millionen Franken humanitärer Hilfe für 1997 wurden 72,2 Millionen Franken über die internationalen Organisationen (davon 28 Millionen für das UNHCR und 26 Millionen für das Welternährungsprogramm - WEP), 81 Millionen über das IKRK und 44 Millionen über die schweizerischen Hilfswerke geleitet. Die direkten Einsätze des Schweizerischen Katastrophenhilfekorps beliefen sich auf 25 Millionen Franken ${ }^{6}$. Graphik 6 gibt die Aufteilung der humanitären Hilfe des Bundes wieder.

Tabelle 21 zeigt die Entwicklung der Beiträge der Schweiz an die wichtigsten multilateralen Organisationen, die im Bereich der humanitären Hilfe tätig sind. Die Koordinierung der humanitären Einsätze der Vereinten Nationen, welche früher die Aufgabe der UN-Abteilung für humanitäre Angelegenheiten (DHA, mit Sitz in Genf) war, wurde 1998 vom neuen UN-Büro für die Koordination humanitärer Angelegenheiten (mit Sitz in New York und einer Vertretung in Genf) übernommen. Die Schweiz ist einer der wichtigsten Geldgeber dieses Koordinationsorgans (mit 6,1 Millionen Franken 1997). Von 1992 bis 1997 waren die UN-Beiträge der Schweiz wie folgt verteilt: Das UNHCR erhielt durchschnittlich $40 \%$ der finanziellen Mittel der Schweiz, das Welternährungsprogramm 39\%, die UNRWA 12\%, die Abteilung für humanitäre Angelegenheiten 4\% (und 5\% für verschiedene Organisationen).

6. Siehe die von der DEZA 1998 anlässlich des 25. Jahrestages der Gründung des SKH herausgebrachte Broschüre «Schweizerisches Katastrophenhilfekorps (SKH)». 


\section{Graphik Nr. 6: Humanitäre Hilfe des Bundes - Aufteilung nach Einsatzarten}

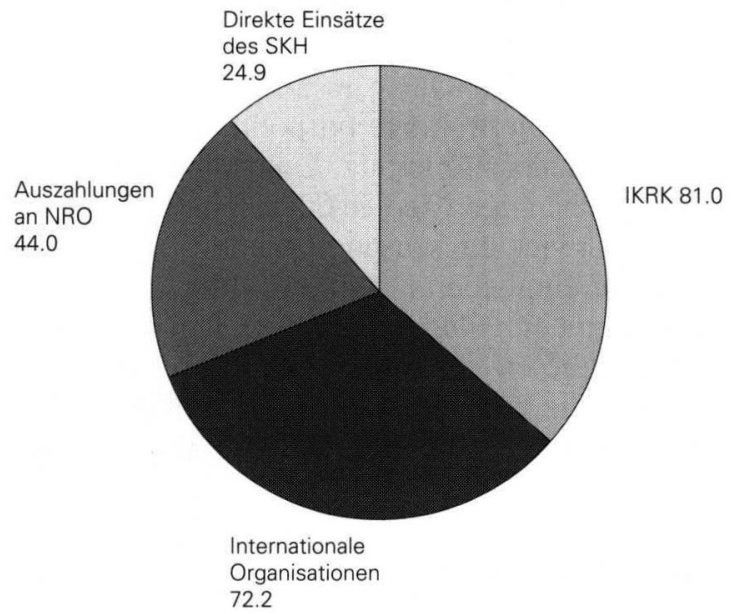

Quelle: DEZA, BAWI, Internationale Zusammenarbeit der Schweiz, Jahresbericht 1997 (Broschüre Humanitäre Hilfe), Bern, 1998.

Tabelle Nr. 21: Beiträge des Bundes an die in der humanitären Hilfe tätigen internationalen Organisationen 1993-1997, in Millionen Franken

\begin{tabular}{lrrrrr}
\hline & $\mathbf{1 9 9 3}$ & $\mathbf{1 9 9 4}$ & $\mathbf{1 9 9 5}$ & $\mathbf{1 9 9 6}$ & $\mathbf{1 9 9 7}$ \\
\hline Multilaterale Organisationen & & & & & \\
\hline UNHCR & 43.2 & 31.9 & 28.4 & 29.1 & 27.4 \\
\hline WEP & 32.1 & 35.1 & 30.5 & 32.6 & 26.9 \\
\hline DAH (Koordination humanitärer Hilfe) & 1.3 & 1.2 & 2.6 & 5.6 & 6.1 \\
\hline UNRWA & 12.7 & 10.6 & 8.7 & 9.5 & 7.7 \\
\hline $\begin{array}{l}\text { Andere Organisationen } \\
\text { (UNICEF, OIM, usw.) }\end{array}$ & 3.1 & 6.1 & 3.4 & 4.1 & 3.2 \\
$\begin{array}{l}\text { Internationale private Organisationen } \\
\text { IKRK }\end{array}$ & 81.9 & 87.6 & 91.2 & 83.2 & 81.0 \\
\hline
\end{tabular}

Quelle: "Bericht des Bundesrates über das Verhältnis zwischen der Schweiz und der Organisation der Vereinten Nationen (UNO)" vom 1. Juli 1998, Anhang 8 und 9.

Die Nahrungsmittelhilfe betrug 1997 32,3 Millionen Franken. Praktisch die gesamte Hilfe lief über schweizerische private Organisationen (in Höhe von 16,6 Millionen Franken) und über das Welternährungsprogramm. Die Nahrungsmittelhilfe setzt sich hauptsächlich aus Milchpulver, Getreide und Mehl, Mais und Reis zusammen.

In Tabelle 22 sind die Hauptempfängerländer oder -regionen der humanitären Hilfe der Schweiz von 1995 bis 1997 aufgeführt. Diese Hilfe konzentrierte sich auf vier Gebiete: Ex-Jugoslawien, das Gebiet der Grossen Seen (Ruanda und Burundi), das Horn von Afrika und die Kaukasusrepubliken. Noch niemals war die Eidgenossenschaft so intensiv wie in Ex-Jugoslawien interveniert ${ }^{7}$. Von 1991 bis 1997 hat die Schweiz Beiträge von über 1,5 Milliarden Dollar im Zusammenhang mit den Folgen der Konflikte im früheren Jugoslawien aufge-

7. Siehe «Swiss Commitment in former Yugoslavia», DDC, August 1998. Die DEZA-Broschüre beschreibt das Engagement der Schweiz in den Staaten Ex-Jugoslawiens. 
bracht. Im gleichen Zeitraum hat die Schweiz humanitäre Hilfe (durch die DEZA) in Höhe von 123 Millionen Dollar sowie einen Beitrag für den Wiederaufbau in Höhe von 52 Millionen Dollar (DEZA und Bundesamt für Flüchtlinge im Rahmen der Rückkehrhilfe für bosnische Flüchtlinge, für Infrastrukturprojekte) aufgewandt. Die Beiträge für politische Reformen beliefen sich auf 35 Millionen Dollar (Friedensförderung, Unterstützung des Wahlprozesses, Gelbmützen, Hilfe für unabhängige Medien). Nur ein Teil der obigen Beiträge wird in der öffentlichen Entwicklungszusammenarbeit der Schweiz berücksichtigt. Die individuelle Wiedereingliederungshilfe für Flüchtlinge belief sich auf 16 Millionen Dollar (Bundesamt für Flüchtlinge). Die von den NRO unterstützten und finanzierten Projekte beliefen sich auf 67 Millionen Dollar. Hierzu kommen Aufwendungen in der Schweiz von über 1,2 Milliarden Dollar für die Aufnahme von Flüchtlingen in der Schweiz (in der öffentlichen Entwicklungshilfe nicht enthaltener Betrag).

Tabelle Nr. 22: Humanitäre Hilfe des Bundes

(Kumulierte Beträge von 1995 bis 1997)

\begin{tabular}{llc}
\hline & $\begin{array}{l}\text { Empfängerländer oder -regionen } \\
\text { der humanitären Hilfe }\end{array}$ & In Millionen Franken \\
\hline 1. & Staaten Ex-Jugoslawiens & 60.8 \\
\hline 2. & Ruanda/Gebiet der Grossen Seen & 37.8 \\
\hline 3. & Angola & 21.9 \\
\hline 4. & Kaukasusrepubliken* & 17.7 \\
\hline 5. & Nordkorea & 13.4 \\
\hline 6. & Äthiopien & 13.2 \\
\hline 7. & Russland & 11.0 \\
\hline 8. & Sudan & 10.6 \\
\hline 9. & Afghanistan & 8.8 \\
\hline 10. & Kongo (Ex-Zaire) & 8.2 \\
\hline 11. & Somalia & 7.7 \\
\hline 12. & Eritrea & 7.6 \\
\hline 13. & Liberia & 7.5 \\
\hline 14. & Mosambik & 6.8 \\
\hline 15. & Irak & 6.5 \\
\hline & Obige 15 Länder/Regionen & $\mathbf{2 3 9 . 5}$ \\
\hline & Andere Länder & 108.9 \\
\hline & Geographisch nicht aufgeteilt & 216.6 \\
\hline & Multilaterale humanitäre Hilfe & 132.3 \\
\hline & Kumulierter Gesamtbetrag der human. Hilfe & $\mathbf{6 9 7 . 3}$ \\
\hline & & \\
\hline
\end{tabular}

Quelle: Jahresbericht 1997 über die internationale Zusammenarbeit, Humanitäre Hilfe, DEZA, BAWI, 1998.

* Georgien, Armenien und Aserbeidschan. 


\subsection{MULTILATERALE HILFE UND INTERNATIONALE ZUSAMMENARBEIT}

\section{$\square$ Bericht über das Verhältnis zwischen der Schweiz und der UNO}

In Erfüllung des (von 84 Abgeordneten mitunterzeichneten) Postulates von Nationalrat Andreas Gross hat der Bundesrat im Juli 1998 seinen Bericht über das Verhältnis zwischen der Schweiz und der Organisation der Vereinten Nationen $(\mathrm{UNO})^{8}$ vorgelegt. Der Bericht fasst die Ziele und die Struktur der UNO sowie die Weiterentwicklung der Beziehungen der Schweiz mit der UNO zusammen.

Die Schweiz misst der multilateralen Abstimmung grosse Bedeutung bei. Die Ziele des Bundesrates, die in seinem Bericht von 1993 über die Aussenpolitik der Schweiz in den 90er Jahren aufgeführt sind, stimmen mit den Zielen der UNO überein". Die Befürworter eines Beitritts der Schweiz zur UNO sind der Ansicht, dass sich die internationale Lage seit 1986, als das Schweizer Volk diesen Beitritt abgelehnt hatte, vollkommen verändert hat. Die Unterschriftensammlung für eine neue Initiative zugunsten des UNO-Beitritts der Schweiz hat im September 1998 begonnen (100'000 Unterschriften sind bis März 2000 erforderlich). Die Initiative wird von einer Gruppe politischer Persönlichkeiten verschiedener Parteien sowie von dreissig NRO lanciert. Der Bundesrat hat zudem im Dezember 1998 angekündigt, dass er den UNO-Beitritt spätestens im Jahr 2003 vorschlagen werde.

\section{$\square$ Bedeutung der multilateralen Abstimmung}

Für den Bundesrat sind das Ende des Kalten Krieges, die Globalisierung, die Interdependenz zwischen den Ländern, das Bestehen komplexer Probleme von globaler Tragweite (zum Beispiel Umwelt, Friedenserhaltung, Migrationen) Faktoren, welche die multilaterale Zusammenarbeit noch wichtiger als früher machen. Die internationalen Debatten, die in der Vergangenheit von den ideologischen Ost-West- und Nord-Süd-Konflikten beherrscht waren, haben sich in den letzten Jahren auf eine vollständigere Erfassung der Probleme hin orientiert, insbesondere mit einer Reihe grosser Weltkonferenzen zur Prüfung globaler Themen: Rechte des Kindes 1990 in New York, Umwelt und Entwicklung 1992 in Rio, Menschenrechte 1993 in Wien, Bevölkerung 1994 in Kairo, Weltkonferenz über Frauen 1995 in Peking und Gipfel für soziale Entwicklung 1995 in Kopenhagen, Habitat II 1996 in Istanbul und Welternährungsgipfel 1996 in Rom. Die Schweiz hat an all diesen Grosskonferenzen teilgenommen, mit Delegationen aus Vertretern und Vertreterinnen der Bundesverwaltung, aber auch aus Akteuren der Zivilgesellschaft (wobei diese Akteure in die Vorbereitung, die Arbeiten und den Folgeprozess der Konferenzen mit einbezogen wurden). Obwohl die auf diesen Konferenzen verabschiedeten Aktionsprogramme keinen bindenden Charakter haben und nicht den ursprünglichen Erwartungen entsprechen, stellen sie dennoch Referenzdokumente dar. Der Bericht des Bundesrates weist darauf hin, dass die oft gemischte Bilanz weniger auf den Inhalt der

8. «Bericht des Bundesrates über das Verhältnis zwischen der Schweiz und der Organisation der Vereinten Nationen (UNO)» vom 1. Juli 1998, Bundesblatt, Nr. 46, 24. November 1998 (Botschaft Nr. 98.051).

9. Der Bericht des Bundesrates führte fünf Schwerpunkte auf: Wahrung und Förderung von Sicherheit und Frieden; Förderung von Menschenrechten, Demokratie und Rechtsstaat; Förderung der Wohlfahrt; Abbau sozialer Gegensätze; Schutz der natürlichen Lebensgrundlagen. 
Dokumente selbst, als auf den mangelnden politischen Willen zurückzuführen ist, sie in den Mitgliedsstaaten rigoros umzusetzen. Einige Konferenzen haben jedoch zur Annahme bindender internationaler Abkommen geführt (die beiden Übereinkommen über Klimaänderungen und über biologische Vielfalt), wie auch zu neuen internationalen Instrumenten oder neuen Strukturen (zum Beispiel der Posten des Hochkommissars der Vereinten Nationen für Menschenrechte).

\section{$\square$ Beteiligung der Schweiz an den multilateralen Institutionen}

Obwohl die Schweiz nicht Mitglied der UNO ist, beteiligt sie sich finanziell an vielen multilateralen Programmen und ist Vollmitglied aller UN-Sonderorganisationen. Das folgende Schema führt die Organisationen des Systems der Vereinten Nationen auf, denen die Schweiz als Mitglied angehört (in fetter Kursivschrift) oder die sie finanziell unterstützt (mit ** gekennzeichnet). Die Schweiz beteiligt sich an den meisten operationellen Tätigkeiten der Vereinten Nationen auf den Gebieten Friedenserhaltung, Menschenrechte, Entwicklungszusammenarbeit, humanitäre Aktionen und Umwelt. Ein Drittel der gesamten humanitären Hilfe des Bundes läuft über multilaterale Organisationen, die im humanitären Bereich tätig sind (und ein weiteres Drittel über das IKRK). Die multilaterale Hilfe macht auch ein Drittel der gesamten längerfristigen Entwicklungszusammenarbeit der Eidgenossenschaft aus.

Der Beobachterstatus bei der UNO erweist sich nach Ansicht des Bundesrates zunehmend als unbefriedigend. Die Schweiz gehört zu den Ländern, die am meisten finanzielle Beiträge leisten, aber in gewissen Beschlussorganen nicht vertreten sein können. Wenn die Schweiz beispielsweise auf der Generalversammlung der Vereinten Nationen das Wort ergreifen will, muss sie sich einem komplexen Verfahren unterziehen, um sicherzustellen, dass kein Staat sich dem widersetzt. Das politische Gewicht der Staaten oder Bewegungen mit Beobachterstatus ist gering (Schweiz, Heiliger Stuhl, Pazifikinseln Tonga und Nauru, PLO). Die übrigen Beobachter sind internationale private Organisationen oder NRO (darunter das IKRK und die Internationale Föderation der Rotkreuz- und Rothalbmondgesellschaften). Die Schweiz kann jedoch in mehreren UN-Kommissionen (zum Beispiel in der Kommission für nachhaltige Entwicklung) oder UN-Komitees (UNAIDS) sowie in gewissen Verwaltungsräten der UN-Organisationen (UNDP, UNEP, UNICEF, UNFPA, UNHCR) als Vollmitglied gewählt werden.

Nimmt man das Jahr 1998 als Berechnungsgrundlage, so würde ein Beitritt der Schweiz zur UNO eine gesamte Mehrbelastung von nur rund 35,7 Millionen Franken ausmachen (13,2 Millionen Franken zusätzliche Beiträge zum ordentlichen Haushalt der UNO und 22,5 Millionen Franken Pflichtbeiträge zu den friedenserhaltenden Aktionen). Hierbei handelt es sich um zusätzliche zu entrichtende Beträge, da die Schweiz bereits einen Teil des ordentlichen UN-Haushalts finanziert (4,9 Millionen Franken 1996) und einen spezifischen (freiwilligen) Beitrag zu den friedenserhaltenden Aktionen (13,4 Millionen Franken 1997) entrichtet. Somit zahlt die Schweiz derzeit zum ordentlichen UN-Haushalt eine Summe, die $30 \%$ des Beitrags entspricht, den sie zu zahlen hätte, wenn sie Vollmitglied wäre. 


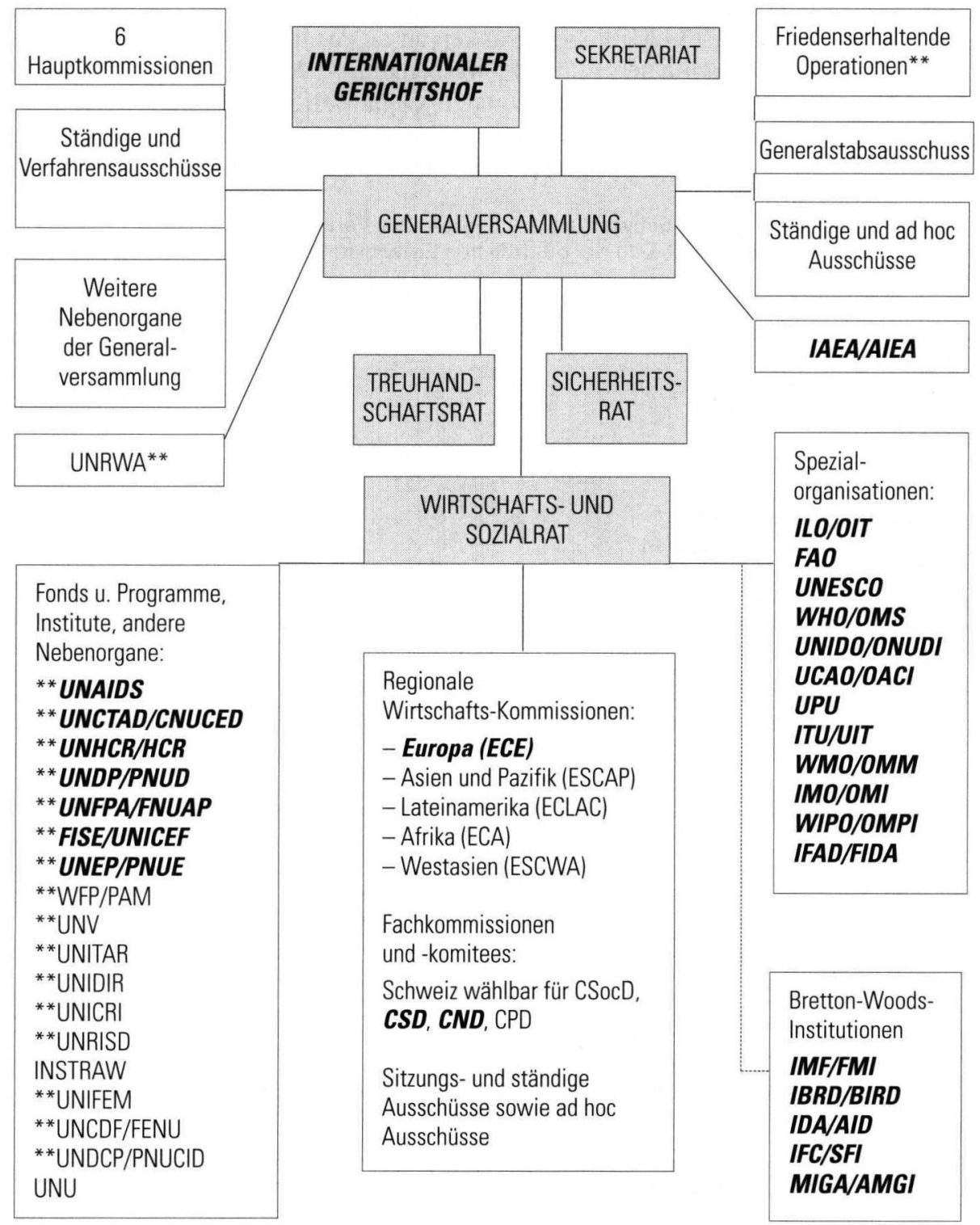

Quelle: «Bericht des Bundesrates über das Verhältnis zwischen der Schweiz und der Organisation der Vereinten Nationen (UNO)" vom 1. Juli 1998, S. 44.

In fetter Kursivschrift: Die Schweiz ist Mitglied oder Vertragspartei (bei Unterorganen: Mitglied des Lenkungsorgans).

*Bezüglich der in diesem Schema verwendeten Abkürzungen verweisen wir auf die Abkürzungsliste am Ende des Jahrbuchs

**Die Schweiz entrichtet freiwillige Beiträge. 
Die multilaterale Hilfe der Schweiz belief sich 1997 auf 376 Millionen Franken. Der Anteil der multilateralen Hilfe an der gesamten öffentlichen Entwicklungshilfe ist in den letzten Jahren (von 23\% 1993 auf 31\% 1997) konstant gestiegen. 1997 hat die Schweiz 139 Millionen Franken an die Weltbankgruppe, 54 Millionen Franken an die regionalen Entwicklungsbanken, 71 Millionen Franken an in der humanitären Hilfe tätige internationalen Organisationen (ohne IKRK) und 121 Millionen Franken an andere UN-Organe gezahlt. Die nachstehende Tabelle zeigt die Entwicklung der Beiträge der Schweiz an das UN-System seit 1993.

Tabelle Nr. 23: Beiträge der Schweiz an das System der Vereinten Nationen 1993-1997 in Mio. Franken (in der öffentlichen Entwicklungshilfe enthaltene Beträge)

\begin{tabular}{|c|c|c|c|c|c|}
\hline & 1993 & 1994 & 1995 & 1996 & 1997 \\
\hline Weltbankgruppe & 133.0 & 151.3 & 146.9 & 183.0 & 138.9 \\
\hline Regionale Entwicklungsbanken & 51.8 & 63.6 & 53.1 & 50.1 & 54.0 \\
\hline $\begin{array}{l}\text { In der Entwicklungszusammenarbeit } \\
\text { tätige UN-Organisationen }\end{array}$ & 148.6 & 149.7 & 135.3 & 131.1 & 120.6 \\
\hline $\begin{array}{l}\text { In der humanitären Hilfe } \\
\text { tätige UN-Organisationen }\end{array}$ & 92.3 & 85.0 & 73.6 & 80.9 & 71.3 \\
\hline $\begin{array}{l}\text { Gesamtbeiträge } \\
\text { an die Vereinten Nationen }\end{array}$ & 425.7 & 449.6 & 408.9 & 445.1 & 384.8 \\
\hline $\begin{array}{l}\text { Gesamtbetrag der öffentlichen } \\
\text { Entwicklungshilfe }\end{array}$ & 1221.6 & 1317.4 & 1269.1 & 1266.1 & 1217.0 \\
\hline $\begin{array}{l}\text { Davon unter multilateraler Hilfe } \\
\text { verbuchte öffentliche Entwicklungshilfe }\end{array}$ & 284.0 & 332.3 & 348.0 & 372.7 & 376.0 \\
\hline $\begin{array}{l}\text { Multilaterale Hilfe } \\
\text { in \% der öff. Entwicklungshilfe }\end{array}$ & 23.25 & 25.22 & 27.42 & 29.44 & 30.90 \\
\hline
\end{tabular}

Quelle: "Bericht des Bundesrates über das Verhältnis zwischen der Schweiz und der Organisation der Vereinten Nationen (UNO)" vom 1. Juli 1998, Anhang 8 und 9.

\section{Finanzielle Beiträge der Schweiz und in der öffentlichen Entwicklungshilfe enthaltener Anteil}

Es ist zu vermerken, dass die über die internationalen Organisationen laufende Hilfe der Schweiz (470 Millionen Franken, ohne IKRK) höher ist als der veröffentlichte Betrag der multilateralen Hilfe (376 Millionen Franken 1997). Dies aus folgenden drei Gründen:

- Die Beiträge zur Finanzierung einzelner Projekte bestimmter internationaler Organisationen werden unter der bilateralen Hilfe verbucht, wenn das Geberland eine direkte Kontrolle über die Projektwahl und die Mittelverwendung behält (assoziierte Hilfe und Kofinanzierungen). Die Hilfe ist multilateral, wenn die Beiträge der verschiedenen Geberländer zu einem einzigen Betrag zusammengelegt werden und die Projekte von den Beschlussgremien der multilateralen Organisation ausgewählt werden. Die gesamte internationale Zusammenarbeit umfasst somit die eigentliche multilaterale Hilfe (allgemeine Beiträge an eine Organisation) und die sogenannte «multibilaterale» Hilfe mit Beiträgen, die für spezifische, von der Schweiz ausgewählte Programme bereitgestellt werden.

- Die Beiträge an die internationalen privaten Organisationen, wie das IKRK, werden unter der bilateralen Hilfe verbucht (diese Zahlungen sind in der obigen Tabelle nicht enthalten). 
- Die Beiträge, die unter der öffentlichen Entwicklungshilfe verbucht werden können, machen nur einen Teil der Beiträge der Schweiz an die Vereinten Nationen und andere internationale Organisationen aus. Nach den Richtlinien der OECD kann ein Beitrag nur unter der multilateralen öffentlichen Entwicklungshilfe verbucht werden, wenn die Empfängerinstitution die Gesamtheit oder einen Teil ihrer Tätigkeit der Entwicklung und den Empfängerländern der Hilfe widmet. Die Beiträge an die multilateralen Organisationen, die nur einen Teil ihrer Tätigkeit der Entwicklung widmen, werden in der öffentlichen Entwicklungshilfe nur bis in Höhe des Anteils ihrer Arbeit, die ihrer Entwicklungstätigkeit entspricht, berücksichtigt. Das DAC überarbeitet periodisch die Liste der Organisationen, für die die Beiträge (gänzlich oder teilweise) unter der öffentlichen Entwicklungshilfe verbucht werden können. Zum Beispiel können seit 1997 84\% der Beiträge an den Internationalen Umweltfonds in der öffentlichen Entwicklungshilfe berücksichtigt werden. Drei Viertel der ordentlichen Beiträge an die WHO und die Gesamtheit der Beiträge an die Spezialprogramme der WHO können als öffentliche Entwicklungshilfe gebucht werden. Die ordentlichen Beiträge an folgende Organisationen können nur teilweise unter der öffentlichen Entwicklungshilfe verbucht werden: WIPO, FAO, IAO, WMO, UPU, ITU, UNESCO.

Unter Berücksichtigung der obigen Angaben läuft rund die Hälfte der öffentlichen Entwicklungshilfe der Schweiz über eine internationale Organisation.

\section{$\square$ Wirtschaftliche Bedeutung des internationalen Genf}

Die wirtschaftlichen Auswirkungen der Präsenz der internationalen Organisationen in Genf sind schwer zu bewerten. Der Bericht des Bundesrates führt hierzu einige Schätzungen $\mathrm{an}^{10}$. Über zwanzig internationale zwischenstaatliche Organisationen und über 120 internationale Nichtregierungsorganisationen haben sich in Genf niedergelassen. 140 Staaten sind in Genf durch eine Ständige Mission vertreten. Die Anwesenheit der internationalen Organisationen zieht den permanenten Aufenthalt von über 30'000 Personen in Genf (und Umgebung) ${ }^{11}$ sowie den temporären Aufenthalt von rund 90'000 Personen im Jahr anlässlich der verschiedenen Konferenzen nach sich. In Genf entfällt mehr als ein Arbeitsplatz von zehn auf den internationalen Sektor, und die Organisationen führen zu einem Auftragsvolumen an Gütern und Dienstleistungen, das auf rund 1,5 Milliarden Franken geschätzt wird. Die Schweiz gehört zu den Ländern, welche die grössten Anteile am Auftragsvolumen der UNO erhalten haben. Die UNO und die WHO kaufen zum Beispiel über die Hälfte der Weltproduktion an Impfstoffen auf (woraus auch die schweizerische Pharmaindustrie Nutzen zieht). Der jährliche wirtschaftliche Beitrag des internationalen Genf wird im Bericht auf insgesamt 2,5 bis 3 Milliarden Franken geschätzt. 55'000 Schweizer und Schweizerinnen arbeiten im UN-System, davon 31'000 in den Sonderorganisationen. Der Wettbewerb zur Aufnahme von internationalen Konferenzen oder von Sekretariaten internationaler Organisationen hat sich verstärkt, da andere Städte ihre Aufnahmebereitschaft bekunden. Genf hat in den letzten Jahren den Sitz der WTO zugesprochen bekommen. Hingegen wurde Bonn das Sekretariat des Rahmenabkommens über Klimaänderungen und das Sekretariat

10. Die Zahlen in diesem Absatz sind dem «Bericht des Bundesrates über das Verhältnis zwischen der Schweiz und der Organisation der Vereinten Nationen (UNO)» vom 1. Juli 1998 entnommen.

11. Internationale Beamte und Diplomaten mit ihren Familien und Hausangestellten. 
des Abkommens über die Wüstenbildung zugewiesen. Der Sitz des Übereinkommens über die biologische Vielfalt wurde in Montreal, derjenige der Organisation für das Verbot chemischer Waffen in Den Haag errichtet.

@ JSDW 1998, Kapitel 5.1., Organisation der Vereinten Nationen.

\subsection{REGIONALE ENTWICKLUNGSBANKEN (ASIATISCHE, INTERAMERIKANISCHE UND AFRIKANISCHE ENTWICKLUNGSBANK)}

\section{$\square$ Beteiligung der Schweiz an der Arbeit der regionalen Entwicklungsbanken}

Die Schweiz ist seit 1967 Mitglied der Asiatischen Entwicklungsbank und gehört den beiden anderen Banken seit deren Öffnung für nichtregionale Länder (das heisst der Interamerikanischen Entwicklungsbank seit 1976 und der Afrikanischen Entwicklungsbank seit 1982) an. Der am 19. Dezember 1995 in Kraft getretene Bundesbeschluss über die Beteiligung der Schweiz an der Kapitalerhöhung der regionalen Entwicklungsbanken bildet die Rechtsgrundlage für das Engagement der Schweiz (siehe Jahrbuch 1996 zum Inhalt der Botschaft). Der betreffende Rahmenkredit von 800 Millionen Franken wurde für die Beteiligung der Schweiz an der Kapitalerhöhung der Interamerikanischen, der Asiatischen und der Afrikanischen Entwicklungsbank, der Interamerikanischen Investitionsgesellschaft sowie der Multilateralen Investitionsgarantie-Agentur (MIGA) eröffnet. Von den 800 Millionen Franken werden nur 45 Millionen effektiv eingezahlt, der Rest bildet Garantiekapital. Die Beiträge der Schweiz an die regionalen Entwicklungsbanken und ihre Spezialfonds beliefen sich 1997 auf 54 Millionen Franken.

\section{Allgemeine Funktionsweise der Entwicklungsbanken}

Die regionalen Entwicklungsbanken sind multilaterale Finanzinstitutionen, deren Ziel es ist, Projekte und Programme zur Entwicklungsförderung durch die Vergabe von Darlehen und die Gewährung technischer Hilfe zu unterstützen. Die Finanzmittel der regionalen Entwicklungsbanken setzen sich aus dem Aktiengrundkapital, den Anleihen auf dem internationalen Kapitalmarkt, den Beiträgen der Geberländer an die Entwicklungsfonds und den Erträgen aus kurzfristigen Anlagen zusammen. Die regionalen Entwicklungsbanken vergeben zwei Arten von Krediten, Kredite zu Marktbedingungen für die wohlhabendsten Länder der Region und Kredite zu günstigen Zinssätzen für die ärmsten Länder, über die Entwicklungsfonds jeder der Banken (konzessionelle Fonds). Dank ihres breit gestreuten Kapitals und der Garantien der Mitgliedsländer können die regionalen Entwicklungsbanken auf den internationalen Märkten die zusätzlichen Mittel zur Finanzierung prioritärer Investitionen im Entwicklungsbereich aufbringen. Die Mitglieder der regionalen Entwicklungsbanken sind in zwei grosse Gruppen aufgeteilt: die Länder des betreffenden Kontinents (regionale Länder) und gewisse Länder anderer Regionen (vor allem Industrieländer), die einen Teil des Grundkapitals besitzen und die regionalen Fonds für die ärmsten Länder finanzieren. Dies erklärt das Interesse der Industrieländer daran, diese Institutionen nicht nur zu finanzieren, sondern auch an den Beschlüssen teilzunehmen und die Geschäftsführung der Banken sowie ihre Kreditverwendungspolitik zu verfolgen.

Die periodischen Verhandlungen über die Kapitalerhöhung der Banken und die Wiederauffüllung ihrer Entwicklungsfonds sind entscheidende Momente, in denen die Arbeitsschwerpunkte für die kommenden Jahre festgelegt werden. Die drei regionalen Entwicklungsbanken sehen sich seit einigen Jahren vor die Notwendigkeit gestellt, die Effizienz ihrer operationellen Tätigkeiten (Programme und Projekte) zu verbessern und institutionelle Reformen vorzunehmen. Die regionalen Entwicklungsbanken müssen der "Gouvernanz» in den Staaten des Südens mehr Aufmerksamkeit schenken: Verbesserung der Verwaltungseffizienz des öffentlichen Sektors, Förderung des Engagements des öffentlichen Sektors im sozialen Bereich bei gleichzeitiger Minimierung unproduktiver Ausgaben wie Militärausgaben und Korruptionsbekämpfung. 
Die Leitung der regionalen Entwicklungsbanken erfolgt durch einen Gouverneursrat (in dem jedes Mitgliedsland mit einem Gouverneur und einem Stellvertreter einsitzt) und einen Exekutivrat (mit Exekutivdirektoren, die eine Ländergruppe vertreten). Die schweizerischen Gouverneure sind Vertreter des BAWI, die stellvertretenden Gouverneure Mitarbeiter der DEZA. Die Schweiz bildet mit anderen (nicht den betreffenden Regionen angehörenden) Ländern eine Stimmrechtsgruppe, die im Rotationsverfahren die Vertreter in den Exekutivrat (d.h. einen Exekutivdirektor, einen stellvertretenden Exekutivdirektor, einen Berater und einen Assistenten des Exekutivdirektors) ernennt. Die nachstehende Tabelle fasst einige Merkmale der drei regionalen Entwicklungsbanken zusammen.

Tabelle Nr. 24: Angaben zu drei regionalen Entwicklungsbanken

\begin{tabular}{|c|c|c|c|}
\hline & $\begin{array}{c}\text { Afrikanische } \\
\text { Entwicklungsbank }\end{array}$ & $\begin{array}{c}\text { Asiatische } \\
\text { Entwicklungsbank }\end{array}$ & $\begin{array}{l}\text { Interamerikanische } \\
\text { Entwicklungsbank }\end{array}$ \\
\hline $\begin{array}{l}\text { Mitgliedsländer } \\
\text { Regionale Länder } \\
\text { (Stimmrechte in \%) } \\
\text { Nichtregionale Länder } \\
\text { (Stimmrechte in \%) }\end{array}$ & $\begin{array}{l}53 \text { Länder }(65.8 \%) \\
24 \text { Länder }(34.2 \%)\end{array}$ & $\begin{array}{l}41 \text { Länder (rund 65\%) } \\
16 \text { Länder (rund 35\%) }\end{array}$ & $\begin{array}{c}46 \text { Länder } \\
28 \text { Länder }(87,4 \%, \\
\text { (davon USA: } 31.8 \%) \\
18 \text { Länder }(12.6 \%)\end{array}$ \\
\hline $\begin{array}{l}\text { Kapital der Bank } \\
\text { Letzte Kapitalerhöhung }\end{array}$ & $\begin{array}{c}29 \text { Mia } \$ \\
1998\end{array}$ & $\begin{array}{c}80 \text { Mia } \$ \\
1994\end{array}$ & $\begin{array}{c}80 \text { Mia } \$ \\
1994\end{array}$ \\
\hline $\begin{array}{l}\text { Volumen der } 1997 \text { bewilligten Darlehe } \\
\text { (in Millionen Dollar) }\end{array}$ & 1776.6 & 9414.0 & 6017.3 \\
\hline aus dem ordentlichen Kapital & 771.1 & 7794.4 & 5649.0 \\
\hline $\begin{array}{l}\text { aus dem konzessionellen Fonds } \\
\text { Spezialfonds }\end{array}$ & 1005.5 & 1619.6 & $\begin{array}{r}283.4 \\
84.9\end{array}$ \\
\hline $\begin{array}{l}\text { Letzte Wiederauffüllung des } \\
\text { konzessionellen Entwicklungsfonds }\end{array}$ & 1996 & 1997 & 1994 \\
\hline Betrag und Laufzeit & 3 Mia \$ 1996-1998 & 6.3 Mia \$ 1997-2000 & $\begin{array}{l}2 \text { Mia } \$ 1994-1997 \\
\text { 1.2 Mia } \$ \text { für } 98 \text { und } 99\end{array}$ \\
\hline $\begin{array}{l}\text { Anteil der Schweiz am Kapital der Ban } \\
\text { (und Stimmrechte in \%) }\end{array}$ & $1.27 \%$ & $0.84 \%$ & $0.47 \%$ \\
\hline $\begin{array}{l}\text { Vertretung der Schweiz } \\
\text { im Exekutivrat }\end{array}$ & $\begin{array}{l}1 \text { Assistentin der } \\
\text { Administratorin } \\
97 \text { bis Juni } 99\end{array}$ & $\begin{array}{l}1 \text { Stellvertretender } \\
\text { Executivdirektor } \\
96 \text { bis Sommer } 98\end{array}$ & $\begin{array}{l}1 \text { Stellvertretender } \\
\text { Executivdirektor } \\
96 \text { bis Sommer } 98\end{array}$ \\
\hline
\end{tabular}

Quellen: Jahresberichte der Entwicklungsbanken und BAWI.

\section{$\square$ Afrikanische Entwicklungsbank}

Der Gouverneursrat der Afrikanischen Entwicklungsbank (BAD) setzt sich aus Vertretern von 53 Mitgliedsländern der Region und 24 nichtregionalen Ländern zusammen. Der Gouverneur der Schweiz bei der BAD (und im Gouverneursrat des Fonds) ist Botschafter Nicolas Imboden vom BAWI, Henri Philippe Cart von der DEZA ist der stellvertretende Gouverneur. Der Exekutivrat der Bank besteht aus 18 Mitgliedern, von denen zwölf Vertreter von Ländern der Region und sechs Vertreter von Ländern ausserhalb der Region sind. Die Stimmrechtsgruppe beim Exekutivrat setzt sich aus Schweden, Dänemark, Finnland, Norwegen, der Schweiz und Indien zusammen. Nach dem Rotationssystem bekleidet Finnland die Exekutivdirektorstelle für diese Stimmrechtsgruppe bis 2001 (eine Schweizerin ist Assistentin der Administratorin bis zum Sommer 1999). 


\section{Reformen der Funktionsweise der Bank}

Die Schweiz verfolgt aufmerksam die Fortschritte bei der Umsetzung der seit 1995 eingeleiteten institutionellen und finanziellen Reformen der Bank ${ }^{12}$. Diese Reformen umfassen vor allem folgende Bereiche: Qualitätsverbesserung der Operationen der Bank und der geförderten Projekte (mit einer besseren Evaluation der Ergebnisse und der Errichtung von Länderstrategien als Rahmen für das dreijährige Darlehensprogramm, einer Leistungssteigerung der ausführenden Organe und einer Verbesserung der Finanzführung), bessere Ausbildung des Personals und Einstellung von qualifizierterem Personal, Reformen der Funktionsweise der Bank.

\section{Operationelle Tätigkeiten}

Die Darlehen der Gruppe der Afrikanischen Entwicklungsbank beliefen sich 1997 auf 1,8 Milliarden Dollar (gegenüber 800 Millionen 1996). Davon entfielen 771 Millionen Dollar auf Darlehen der Bank und etwas über eine Milliarde Dollar auf Geschenke und Darlehen des Afrikanischen Entwicklungsfonds (konzessioneller Fonds für die ärmsten Länder). Die Aktivitäten des Fonds kamen 1994 und 1995 zum Erliegen (keinerlei Darlehen vor der 1996 beschlossenen Wiederauffüllung des FAD), danach beliefen sich die FAD-Darlehen 1996 auf 286 Millionen Dollar (zum Vergleich: in den Jahren 1989 bis 1993 lagen die Darlehen des Fonds zwischen 900 und 1160 Millionen Dollar). Dieser Stillstand der Fondstätigkeit war namentlich auf die schwierigen Verhandlungen über die (1996 bewilligte) Wiederauffüllung des FAD zurückzuführen.

Die Darlehen der Bank gingen vor allem in die Länder Nordafrikas (59\% der Gesamtdarlehen) und nach dem südlichen Afrika (34\%). Die Länder Westafrikas (38\%) und Ostafrikas (30\%) sind die Hauptempfänger des Fonds. Die wichtigsten Bereiche, in denen Darlehen der Bank vergeben wurden, sind die Industrie (40\% der Darlehen), die öffentlichen Dienste (16\%) und der Transport (12\%). Die Landwirtschaft und der soziale Sektor machen 39\% der Darlehen des Fonds aus. Dieser Anteil liegt noch weit hinter dem Ziel von 60\% zurück, das bei den Verhandlungen über die siebte Wiederauffüllung des FAD festgesetzt worden war. Mehrere Mitgliedsländer der BAD, die der Region nicht angehören, aber wichtige Geldgeber des FAD sind, würden es begrüssen, dass die Bank ihre Tätigkeit auf die Armutsbekämpfung konzentriert.

\section{Kapitalerhöhung der Bank}

Die 34. Jahresversammlung der Afrikanischen Entwicklungsbank (BAD) und die 25. Versammlung des Afrikanischen Entwicklungsfonds (FAD) fanden Ende Mai 1998 in Abidjan statt. Nach langwierigen zweijährigen Verhandlungen haben die Gouverneure der BAD ein Abkommen über eine 35\%ige Erhöhung des Kapitals der Bank abgeschlossen, das dadurch von 21 auf 29 Milliarden Dollar aufgestockt wird. Der Anteil der nichtregionalen Länder am Gesamtkapital ist grösser als zuvor (und erhöht sich von einem Drittel auf 40\%). Die Kapitalaufstockung erlaubt es der Bank, ihre Finanzgrundlage zu verstärken (zum Beispiel günstigere Bedingungen auf den internationalen Finanzmärkten zu erlangen). Der strittigste Punkt der Verhandlungen war die Frage der Stimmenverteilung zwischen regionalen und nichtregionalen Ländern bei den Abstimmungen. Die nichtregionalen Länder wollten ihren Einfluss bei der Beschlussfassung verstärken, wogegen einige grosse regionale Länder einen zu starken Verlust des

12. Siehe zum Beispiel den Abschnitt über die Reformen in den regionalen Entwicklungsbanken im «Bericht zur Aussenwirtschaftspolitik 97/1+2» vom 19. Januar 1998, S. 163-165. 
afrikanischen Charakters der Bank befürchteten. Der bei den Abstimmungsregeln erreichte Kompromiss besagt, dass die Beschlüsse im allgemeinen mit einer Zweidrittelmehrheit gefasst werden müssen, dass sie aber ausnahmsweise mit einer 70\%-Mehrheit erfolgen können, wenn ein Exekutivdirektor dies aufgrund der Bedeutung des Beschlusses für die von ihm vertretenen Länder ausdrücklich beantragt. Mehrere nichtregionale Länder brachten ihre Unzufriedenheit über den erreichten Kompromiss zum Ausdruck, da die Abstimmungsregeln zu sehr auf einer Konfrontations- und Sperrminoritätslogik beruhten, während man eine Partnerschaft unter den Ländern aufbauen und einen Konsens zu gewissen Fragen suchen sollte. Im Anschluss an das obige Abkommen haben die nichtregionalen Länder die Verhandlung zur Wiederauffüllung des Afrikanischen Entwicklungsfonds (achte Wiederauffüllung des FAD) aufgenommen.

\section{Verringerung der Verschuldung}

Die Schweiz hat im Dezember 1998 mit dem Afrikanischen Entwicklungsfonds ein Abkommen über einen Beitrag von 8 Millionen Franken zum zusätzlichen Finanzierungsmechanismus des FAD unterzeichnet. Dieser Mechanismus wurde 1997 geschaffen, um den einkommensschwachen regionalen Mitgliedsländern zu helfen, die Rückstände bei den Zinszahlungen auf die Kredite der BAD zu begleichen. Die Massnahme ist Teil der Programme zur Reduzierung der externen Schuld der ärmsten Länder (zum Beispiel mit der HIPC-Initiative). Dieser Mechanismus des FAD kam 1998 zwölf Ländern mit einem Gesamtbetrag von 59 Millionen Dollar zugute.

\section{$\square$ Asiatische Entwicklungsbank}

Die 31. Jahresversammlung der Asiatischen Entwicklungsbank (ADB) fand vom 29. April bis 1. Mai 1998 in Genf statt. Es war das erste Mal, dass die Jahresversammlung einer regionalen Entwicklungsbank von der Schweiz aufgenommen wurde. Die schweizerische Delegation setzte sich aus den Bundesräten Flavio Cotti und Pascal Couchepin, Botschafter Nicolas Imboden (BAWI), zwei weiteren Vertretern des BAWI und einem Vertreter der DEZA zusammen. Nicolas Imboden hat den Vorsitz des Gouverneursrates vom Sommer 1997 bis zum Sommer 1998 geführt. Auf der Versammlung wurden von der Asiatischen Entwicklungsbank, Universitätsinstituten, internationalen Organisationen und Banken gemeinsam mehrere Seminarien (zum Beispiel über die asiatische Finanzkrise, die soziale Entwicklung, das Gesundheitswesen, die Telekommunikationen und den städtischen Sektor) veranstaltet. Tadschikistan ist der BAD 1998 als 57. Mitgliedsland beigetreten. Die Schweiz hat den Beitritt dieses Landes erleichtert, indem sie seinen Anteil am Kapital der Bank finanzierte (Tadschikistan gehört zur Stimmrechtsgruppe der Schweiz bei IWF und Weltbank).

In seiner Eröffnungsansprache vor der Jahresversammlung hob Bundesrat Flavio Cotti die Bedeutung einer guten Regierungsführung für eine nachhaltige Entwicklung zugunsten der gesamten Gesellschaft hervor. «Die wesentlichen Grundlagen eines solchen Prozesses sind der Rechtsstaat, eine transparente öffentliche Verwaltung, die Justiz, eine möglichst gerechte Einkommensverteilung, ausgewogene Stadt-Land-Beziehungen, Institutionen, durch welche die Bürger und Bürgerinnen sich ausdrücken können sowie freie und verantwortliche Informationsmittel» ${ }^{13}$. 
Am Rande der Versammlung kündigte die Schweiz einen Beitrag von 2 Millionen Dollar für die Einsetzung eines Treuhandfonds zur Förderung des Privatsektors an. Bundesrat Pascal Couchepin und DEZA-Direktor Walter Fust haben ferner mit einem Dutzend Delegationen asiatischer Länder bilaterale Gespräche geführt.

\section{Interventionen der $A D B$ nach der Asienkrise}

Die Diskussionen der Jahresversammlung wurden von der Asienkrise beherrscht. Um dieser Krise entgegenzuwirken und Rettungsaktionen zu finanzieren, hat sich die Asiatische Entwicklungsbank massiv an der Seite der Bretton-WoodsInstitutionen engagiert. Die Verpflichtungen der ADB haben sich dadurch fast verdoppelt, indem sie von 5,5 Milliarden Dollar 1996 auf 9,4 Milliarden 1997 anstiegen, davon 7,8 Milliarden Dollar für Darlehen aus dem ordentlichen Kapital und 1,6 Milliarden für Darlehen des konzessionellen Asiatischen Entwicklungsfonds (ADF). Die finanziellen Verpflichtungen richteten sich vor allem auf die Unterstützung der Reformen im Finanz- und Bankensektor der von der Krise betroffenen Länder (die Reform der Finanzmärkte absorbierte die Hälfte der Darlehen der Bank, einschliesslich ADF). Die Hauptempfängerländer der Darlehen aus dem ordentlichen Kapital waren 1997 Südkorea (mit einem Rekordkredit von 4 Milliarden Dollar für den Finanzsektor ${ }^{14}$, das heisst das grösste seit der Gründung der Bank gewährte Kreditprogramm), Indonesien (Kredite für 1,1 Milliarden Dollar), China, Indien und Thailand (550 Millionen). Die Krisenstaaten, deren Beträge oben aufgeführt sind, haben allein über $72 \%$ der Darlehen aus dem ordentlichen Kapital erhalten. Die Hauptempfängerländer der Mittel des Asiatischen Entwicklungsfonds waren Bangladesch (420 Millionen Dollar), Vietnam (360 Millionen Dollar) und Pakistan (251 Millionen).

Wenn auch viele Länder auf dem Jahrestreffen betonten, dass die Reaktion der Asiatischen Entwicklungsbank zur Eindämmung der Krise rasch und willkommen war, so äusserten einige Länder jedoch ihre Beunruhigung über den Umfang der für diese Nothilfeaktion eingesetzten Mittel im Verhältnis zur Hauptaufgabe der Bank, die eine Unterstützung der langfristigen Entwicklung sein sollte. Mehrere Länder haben insbesondere mit Nachdruck auf die Bedeutung des Auftrags der Bank bei der Armutsbekämpfung hingewiesen. Die 1997 gewährten umfangreichen Darlehen gingen mit einer Aufstockung der Reserven der Bank und folglich mit einer Verringerung der für Aktivitäten der technischen Hilfe oder zur Finanzierung des Asiatischen Entwicklungsfonds verfügbaren Mittel einher (der Fonds wird gemeinsam von den Geberländern und durch Beiträge aus dem Kapitalnettoertrag der Bank finanziert).

\section{$\square$ Interamerikanische Entwicklungsbank}

Die Interamerikanische Entwicklungsbank (IDB) beteiligt sich an der Finanzierung von Programmen und Projekten der Mitgliedsländer (alle lateinamerikanischen Länder ausser Kuba). Sie kann auch Darlehen ohne staatliche Garantie für den privaten Sektor dieser Länder gewähren. Die Interamerikanische Investitionsgesellschaft (IIC) vergibt Darlehen an kleine und mittlere Unternehmen oder Finanzintermediäre. 35 Länder gehören der IIC als Mit-

14. Das Reformprogramm des koreanischen Finanzsektors umfasst Kapazitätsbildungsmassnahmen für den Bankensektor, die Errichtung von Infrastrukturen für den Finanzmarkt sowie Massnahmen zur Verbesserung der Kontrolle der Privatfirmen und der Informationsstandards. 
glieder an (gegenüber 46 Mitgliedsländer der IDB), davon zehn nichtregionale Länder (unter ihnen die Schweiz). Die Jahresversammlungen des Gouverneursrates der IDB und der Interamerikanischen Investitionsgesellschaft fanden im März 1998 in Cartagena de Indias (Kolumbien) statt. Die Jahresversammlung 1999 der beiden Institutionen findet vom 15. bis 17. März dieses Jahres in Paris statt.

\section{Operationelle Tätigkeiten}

Das Gesamtvolumen der von der Bank gewährten Darlehen belief sich 1997 auf 6 Milliarden Dollar (gegenüber 6,7 Milliarden im Vorjahr), davon 5,6 Milliarden Dollar aus dem Kapital der Bank und 283 Millionen aus dem konzessionellen Fonds. Die Hauptempfängerländer der Darlehen aus dem ordentlichen Kapital der Bank sind Brasilien (mit Darlehen von 1,5 Milliarden Dollar 1997), Argentinien (1,2 Milliarden) (auf diese beiden Länder entfallen $48 \%$ des Gesamtdarlehensvolumens), Uruguay, Panama, Kolumbien und Peru. Die Hauptempfängerländer des konzessionellen Fonds (Fonds für Spezialoperationen, FSO) sind Nicaragua, Haiti, Guyana und Ekuador.

\section{Jahresversammlung}

Auf der Jahresversammlung wurde das Projekt «Vision der Bank» geprüft, das eine Partnerschaftsstrategie zwischen den Mitgliedsstaaten für die kommenden Jahre und die Festlegung der Modalitäten einer Anpassung des Mandats der Bank an die neuen wirtschaftlichen Realitäten der Region empfiehlt (neue Produkte, Entwicklung der Arbeit mit der Zivilgesellschaft, Unterstützung des Privatsektors). Die anderen Hauptpunkte auf der Tagesordnung waren die Frage der Finanzierung von Projekten des Privatsektors durch die Bank und das Problem der Finanzmittel für die ärmsten Länder. Im Rahmen der Diskussionen über die Zukunftsvision der Bank erhoben sich Stimmen zugunsten einer Lockerung der Kreditvergaberegeln (vor allem dem Wunsch einiger regionaler Länder entsprechend). Gemäss den bei der achten Kapitalerhöhung der Bank 1994 (IDB-VIII) festgesetzten Schwerpunkten sollten mindestens $40 \%$ der Darlehen den sozialen Bereich (in einem ziemlich weit gefassten Sinne ${ }^{15}$ ) unterstützen und 35\% der Darlehen an die ärmsten Länder der Region vergeben werden $^{16} .1997$ entfielen $43 \%$ der Kredite auf den sozialen Bereich, und die ärmsten Länder machten $27 \%$ der Gesamtkredite der Bank aus. Einige Länder wünschten auch eine Heraufsetzung der Höchstgrenze der Direktkredite für den privaten Sektor (durch eine Erhöhung des gegenwärtigen Anteils von maximal 5\% der Gesamtdarlehen der Bank auf 10\%) sowie eine Erhöhung des Projektanteils, der von der Bank übernommen werden kann. Die Untersuchungen werden fortgesetzt, da bislang noch kein Konsens über die Lockerung dieser Regeln gefunden werden konnte. Die meisten nichtregionalen Länder (die einen Teil des Kapitals der Bank besitzen und zur Finanzierung des konzessionellen Fonds (FSO) beitragen) haben ihr Festhalten an den Zielen, die auf der achten Kapitalerhöhung der Bank (IDB-VIII) festgesetzt worden waren, bekräftigt.

Im Anschluss an die bei den Verhandlungen von IDB-VIII eingegangenen Verpflichtungen hat die Bank 1997 neue Richtlinien in gewissen Bereichen gebilligt: Klassifikation der Darlehen zur Armutsbekämpfung, Strategien im Energie-

15. Der soziale Bereich umfasst folgende Gebiete: Gesundheit und Hygiene, städtische Entwicklung, Bildung, Sozialinvestitionen, Umwelt, Kleinstunternehmen.

16. Die ärmsten und die kleinen Länder, das heisst alle Länder Lateinamerikas ausser Argentinien, Brasilien, Chile, Kolumbien, Mexiko, Peru und Venezuela. 
und Bildungssektor, Unterstützung der HIPC-Initiative, Ausschreibungsverfahren zur Korruptionsbekämpfung, Darlehen ohne staatliche Garantie für den Privatsektor. Die Politik zur Unterstützung der Strukturanpassungsmassnahmen ist zur Zeit in Überarbeitung, namentlich mit dem Vorhaben, Darlehen zur Unterstützung des institutionellen Anpassungsprozesses ${ }^{17}$ in Form von Budgethilfen einzurichten, um gewisse Kosten der Anpassung (nicht durch eine Zahlungsbilanzhilfe, sondern durch Budgetausgaben des Staates) zu decken. Die heikle Frage hierbei ist, eine Finanzierung für Investitionen und nicht für die Deckung von Verwaltungsbudgetdefiziten bereitzustellen.

In den Diskussionen der Plenarsitzung wurde darauf hingewiesen, dass sich hinter den im allgemeinen positiven wirtschaftlichen Leistungen, die in Lateinamerika zu verzeichnen sind, grosse Unterschiede zwischen den Ländern verbergen und dass diese positiven Ergebnisse nicht mit den erwarteten Fortschritten im sozialen Bereich einhergegangen sind. Die sozialen Ungleichheiten und die Armut schreiten trotz des starken Wirtschaftswachstums, des Inflationsrückgangs und der Zunahme der Privatkapitalzuflüsse weiter voran.

Wiederauffüllung des konzessionellen IDB-Fonds (FSO)

Die Verhandlungen über die neunte Wiederauffüllung des Fonds für Spezialoperationen (FSO) sind wegen Meinungsverschiedenheiten zwischen den Geberund Empfängerländern über die Finanzierungsweise (Verwendung bankinterner Mittel oder neue Beiträge der Geberländer) verschoben worden. Der Gouverneursrat hat ein Abkommen für die Jahre 1998 und 1999 abgeschlossen, wobei er noch auf die Ressourcen des Fonds (1,2 Milliarden Dollar) zurückgreift. Das Abkommen erlaubt es, die Darlehen für die ärmsten Länder beizubehalten und zur Initiative für die Reduzierung der Schulden Boliviens und Guyanas (HIPC-Initiative) beizutragen, jedoch bleibt die Frage der konzessionellen Mittel ab dem Jahr 2000 ungelöst. Eine Kapitalerhöhung der Interamerikanischen Investitionsgesellschaft konnte bisher wegen des Widerstands der USA noch nicht beschlossen werden, wogegen die meisten europäischen Länder für eine solche Kapitalerhöhung eintraten.

@ JSDW 1998, Kapitel 10, S. 342-344, betreffend die Kapitalerhöhung der Europäischen Bank für Wiederaufbau und Entwicklung (EBWE) und die Aktivitäten der EBWE.

17. IAL (Institutional Adjustment Lending). 


\section{QUELLEN}

\section{Allgemeine Quellen zur Entwicklungshilfe}

OCDE, Coopération pour le développement. Efforts et politiques des membres du Comité d'aide au développement. Rapport 1997, Paris, OCDE, 1998.

Communiqué de presse, OCDE, 18 juin 1998 «L'aide et les apports de capitaux privés ont diminué en 1997».

\section{Öffentliche Hilfe der Schweiz}

Internationale Zusammenarbeit der Schweiz, Jahresbericht 1997, Bern, DEZA, BAWI, 1998.

DEZA, Förderung der Menschenrechte und Entwicklungszusammenarbeit, Bern, DEZA, 1997 (24 Seiten).

«Bericht des Bundesrates über das Verhältnis zwischen der Schweiz und der Organisation der Vereinten Nationen (UNO) in Erfüllung des Postulates von Nationalrat Andreas Gross vom 18. Juni 1997», vom 1. Juli 1998, Bundesblatt, Nr. 46, 24. November 1998, S. 5242-5307 (Bericht Nr. 98.051).

«Bericht über die internationale Zusammenarbeit der Schweiz mit Ländern des Südens und des Ostens 1986-1995, in Erfüllung des Postulates Nr. 96.3405 Zapfl vom 17. September 1996», vom 6. Mai 1998.

«Bericht über die Aussenwirtschaftspolitik 97/1+2» vom 19. Januar 1998, Bundesblatt, Nr. 9. 10.3 .98 (Botschaft Nr. 97.090).

DEZA/BAWI, Internationale Zusammenarbeit der Schweiz. Jahresbericht 1997 (3 Broschüren über Humanitäre Hilfe, Entwicklungszusammenarbeit und Zusammenarbeit mit Osteuropa) DEZA/BAWI, Bern, 1998.

DEZA/BAWI, Wer arm ist, ist selber schuld! Antworten auf Fragen zur internationalen Zusammenarbeit der Schweiz (Argumente zur Entwicklungszusammenarbeit). Bern, DEZA und BAWI, 1999, (36 Seiten).

DEZA-Pressedienst (erscheint viermal jährlich).

DEZA, Agenda Politik (monatliche Herausgabe).

Newsletter, Kurzinformationen aus der DEZA (erscheint achtmal jährlich).

Schweiz global, Magazin des EDA (erscheint fünfmal jährlich, telefonische Bestellung unter der Nr. 031/322 31 53).

DDC, Politique sectorielle DDC dans le domaine financier, avril 1998, 46 p. (Série Politiques sectorielles de la DDC).

\section{Internet-Adressen und Bestellung von Publikationen}

EDA, Eidgenössisches Departement für auswärtige Angelegenheiten: http://www.dfae.admin.ch - enthält ein ganzes Kapitel über internationale Organisationen: www.dfae.admin.ch/site/f/aussenpolitik/internationale.html

DEZA, Direktion für Entwicklung und Zusammenarbeit: http://www.sdc-gov.ch

SKH, Schweizerisches Katastrophenhilfekorps: http://www.skh.ch

OECD, Entwicklungshilfeausschuss (DAC): http://www.oecd.org/dac/ - über diese Internet-Adresse kann man sich mit den Internet-Seiten der Dienststellen für Entwicklungszusammenarbeit der DAC-Mitgliedsländer verbinden.

Eurofic, Internet-Seite der Europäischen Union über Entwicklungszusammenarbeit: http://www.oneworld.org/eurofic

Bestellung von DEZA-Publikationen: Sektion Medien und Kommunikation der DEZA, 3003 Bern, Fax: 031/324 13 48, E-Mail: info@deza.admin.ch

\section{Humanitäre Hilfe}

DEZA, Eine Welt, Das DEZA-Magazin für Entwicklung und Zusammenarbeit, Nr. 3, September 1998 (Bosnien und der Friede).

DEZA, Schweizerisches Katastrophenhilfekorps (SKH), Broschüre zum 25. Jahrestag der Gründung des SKH, 1998.

DDC, Swiss Commitment in Former Yugoslavia, DDC, Bern, August 1998.

\section{Regionale Entwicklungsbanken}

Asian Development Bank, Annual Report 1997, Manila, Philippines, 1998.

Banque interaméricaine de développement, Rapport annuel 1997, Washington, 1998.

L'AGEFI, 27 mars 1998, 22 avril 1998, 27 avril 1998, 4 mai 1998 (Assemblée annuelle de la Banque asiatique de développement).

Neue Zürcher Zeitung, 19. März 1998, «39. Jahresversammlung der IDB».

\section{INTERNET-ADRESSEN:}

Asiatische Entwicklungsbank: http://www.adb.org

Afrikanische Entwicklungsbank: http://www.afdb.org

Interamerikanische Entwicklungsbank: http://www.iadb.org 